AperTO - Archivio Istituzionale Open Access dell'Università di Torino

\title{
Nonalcoholic fatty liver disease
}

\section{This is a pre print version of the following article:}

Original Citation:

Availability:

This version is available http://hdl.handle.net/2318/1636665

since 2017-05-19T15:35:49Z

Published version:

DOI:10.1038/nrdp.2015.80

Terms of use:

Open Access

Anyone can freely access the full text of works made available as "Open Access". Works made available under a Creative Commons license can be used according to the terms and conditions of said license. Use of all other works requires consent of the right holder (author or publisher) if not exempted from copyright protection by the applicable law. 


\section{Nonalcoholic fatty liver disease}

Elizabeth M. Brunt ${ }^{1}$, Vincent W-S Wong ${ }^{2}$, Valerio Nobili ${ }^{3}$, Christopher P. Day ${ }^{4}$, Silvia Sookoian ${ }^{5}$, Jacquelyn J. Maher ${ }^{6}$, Elisabetta Bugianesi ${ }^{7}$, Claude B. Sirlin ${ }^{8}$, Brent A. Neuschwander-Tetri ${ }^{9}$ and Mary E. Rinella ${ }^{10}$

${ }^{1}$ Department of Pathology and Immunology, Washington University School of Medicine, 660 S. Euclid Avenue, Campus Box 8118, Saint Louis, MO 63110, United States

${ }^{2}$ Department of Medicine and Therapeutics and State Key Laboratory of Digestive Disease, The Chinese University of Hong Kong, Hong Kong

${ }^{3}$ Hepatometabolic Unit, Bambino Gesu Children Hospital, Rome, Italy

${ }^{4}$ Institute of Cellular Medicine, Newcastle University, Newcastle upon Tyne, United Kingdom

${ }^{5}$ Department of Clinical and Molecular Hepatology, Institute of Medical Research A Lanari-IDIM, University of Buenos Aires- National Scientific and Technical Research Council (CONICET), Ciudad Autónoma de Buenos Aires, Argentina

${ }^{6}$ Department of Medicine, University of California, San Francisco, CA 94110, United States

${ }^{7}$ Department of Medical Sciences, University of Torino, Città della Salute e della Scienza, Torino, Italy ${ }^{8}$ Department of Radiology, University of California at San Diego, San Diego, CA, Zip Code, United States

${ }^{9}$ Division of Gastroenterology and Hepatology, Saint Louis University School of Medicine, St. Louis, MO, 63110, United States

${ }^{10}$ Department of Medicine, Northwestern University Feinberg School of Medicine, Chicago, IL 60611, United States

Correspondence to:

E.M.B.

ebrunt@ wustl.edu 
Nonalcoholic fatty liver disease (NAFLD) is a liver disease characterized by excess fat accumulation in the hepatocytes (nonalcoholic fatty liver, NAFL); in up to $40 \%$ of individuals, there are additional findings of portal and lobular inflammation, and hepatocyte injury (nonalcoholic steatohepatitis, NASH). A small percent of patients will develop progressive fibrosis and cirrhosis. Hepatocellular carcinoma and cardiovascular complications are life-threatening co-morbidities of both NAFL and NASH. NAFLD is closely associated with insulin resistance; obesity and metabolic syndrome are common underlying factors. As a consequence, the prevalence of NAFLD is estimated to be $10-40 \%$ in adults worldwide, and it is the most common liver disease in children and adolescents in developed countries. Mechanistic insights into fat accumulation, subsequent hepatocyte injury, the role of the immune system and fibrosis as well as the role of the gut microbiome are unfolding, and genetic and epigenetic factors might explain the considerable inter-individual variation in disease phenotype, severity and progression. To date, no effective medical interventions exist that completely reverse the disease other than lifestyle changes, dietary alterations and possibly, bariatric surgery. However, several strategies targeting pathophysiological processes such as oversupply of fatty acids to the liver, cell injury and inflammation are currently under investigation. Diagnosis of NAFLD, and detecting the lesions of NASH, still depend on the gold-standard but invasive liver biopsy. Several non-invasive strategies are being validated to replace or complement biopsies, especially for follow-up monitoring. 
Nonalcoholic fatty liver disease (NAFLD) has been accepted as a bona fide disease entity in the late $20^{\text {th }}$ century. The disorder is characterized by accumulation of fat (in the form of triglycerides) in hepatocytes ( $>5 \%$ fat content in the liver; referred to as steatosis). In the presence of additional factors, described below, hepatocellular injury and death occur along with lobular and portal inflammation, and the disease is referred to as nonalcoholic steatohepatitis (NASH). Specific sites of collagen deposition and subsequent vascular remodelling, and ultimately cirrhosis may result.

Several histological features of NAFLD and NASH are common to alcoholic fatty liver disease (AFLD), including steatosis, inflammation, hepatocellular ballooning (a type of hepatocyte cell injury), Mallory-Denk bodies (Mallory's hyaline) and fibrosis within the lobules. However, in contrast to the underlying exposure to excess alcohol that initiates and drives AFLD, patients with NAFLD are insulin resistant, usually obese, and are known not to consume excess alcohol. ${ }^{1}$ Interestingly, whereas paediatric NAFLD shares similar metabolic triggers with NAFLD in adults, different patterns of histologic injury may be observed ${ }^{2}$.

Morbidity and mortality in patients with NAFLD are mainly caused by the cardiovascular complications, rather than by the liver disease itself ${ }^{3,4}$. Cirrhosis and hepatocellular carcinoma (HCC) are the most common liver-related causes of morbidity associated with NAFLD, followed by systemic infection $^{5}$. That HCC is documented to occur in the absence of cirrhosis is a concern for clinical care ${ }^{6}$.

Finally, the value of the liver biopsy as a diagnostic tool, although an invasive procedure, continues to be shown by large studies analysing the correlation between long-term outcomes with histopathological evaluation. Advanced fibrosis has been identified as the most significant histologic feature associated with poor outcomes in two studies ${ }^{5,7}$; inflammation is associated with initiation and progression of fibrosis ${ }^{8}$. Both in children and in adults, NASH-related cirrhosis is predicted to become the most common indication for liver transplant ${ }^{9}$. Several non-invasive diagnostic tools are currently being validated and show promise.

In this Primer, we describe the worldwide prevalence of NAFLD in adults and children, highlight genomic and epigenomic changes associated with disease and its progression, as well as the molecular drivers of disease and elaborate on the advances in diagnosis and the current lack of management options and quality of life concerns. Avenues for progress are discussed.

\section{[H1] Epidemiology}

[H2] Adults 
[H3] The United States and Europe. NAFLD is currently the most common cause of abnormal liver function tests in Western countries. According to population studies using ultrasonography or CT imaging, the prevalence of NAFLD is in the range $20-50 \%$ (Table 1). ${ }^{10-13}$ NAFLD prevalence varies markedly between ethnic groups. An urban population study in the United States using protonmagnetic resonance spectroscopy $\left({ }^{1} \mathrm{H}-\mathrm{MRS}\right)$ showed that the prevalence of hepatic steatosis was $45 \%$ in Hispanic, $33 \%$ in white, and $24 \%$ in black populations. ${ }^{14}$ These variations can be explained by differences in lifestyle, prevalence of metabolic syndrome and genetics, such as polymorphism of the patatin-like phospholipase domain-containing protein 3 (PNPLA3) gene, which encodes a lipase that mediates triacylglycerol hydrolysis in adipocytes. (Figure 1). NAFLD is strongly associated with metabolic disorders. Not surprisingly, fatty liver has been reported in $40-80 \%$ of patients with type 2 diabetes mellitus and $30-90 \%$ of obese patients. ${ }^{15,16}$

Since imaging can only detect fatty liver but not necroinflammation or fibrosis, the prevalence of NASH and the related liver fibrosis in the population is unclear. Histological analyses suggest that 6$55 \%$ of patients with NAFLD have NASH, depending on the patient inclusion criteria and the definition of NASH. ${ }^{17-19}$ Nonetheless, patients undergoing liver biopsy are highly selected and are not representative for a community. In a study of 328 unselected patients attending an out-patient clinic in the Southwest of the US, $46 \%$ had NAFLD based on patient history and ultrasonography findings. ${ }^{12}$ Of those with NAFLD, 134 (of 156 in total) underwent liver biopsy, of whom 40 were found to have $\mathrm{NASH}$, suggesting an NASH prevalence of $12 \%$ in the general population. Furthermore, using a score validated against liver histology, a Finnish group estimated the population prevalence of NASH to be around $5 \% .{ }^{20}$

With ongoing necroinflammation and liver injury, patients with NASH will develop progressive liver fibrosis and, finally, cirrhosis. Advanced fibrosis or cirrhosis due to NASH has a similar mortality than when these features are caused by chronic hepatitis $C$ virus infection and advanced liver disease. The annual incidence of HCC in patients with NASH-related cirrhosis is $1-2 \%{ }^{21}$. In the United States, NASH has already become the second-leading aetiology of liver disease requiring liver transplantation in adults. ${ }^{22}$ Importantly, the number of patients with NASH on the transplantation waiting list increased by 170\% from 2004 to 2013, the largest absolute and relative increase compared with other aetiologies. Hepatic complications represent the third most common cause of death in patients with NAFLD or NASH, after cardiovascular events and malignancies. ${ }^{4}$ 
[H3] Asia, Africa and Pacific Islands. The epidemiology of NAFLD in Asia-Pacific and African countries is shaped by significant differences in lifestyle and economic growth among countries and between urban and rural areas within the same country. For example, in China the prevalence of NAFLD is higher in coastal and urban areas (up to $30 \%$ ) than in inland and rural areas (around $1 \%$ ). ${ }^{23}$ In urban areas, the prevalence of NAFLD is around $15-30 \%$ in Asia-Pacific countries and $10-20 \%$ in Africa.

Compared with Western countries, the increase in NAFLD prevalence over time is more marked in Asia and the Pacific areas, probably reflecting rapid lifestyle changes. Studies from Japan and China recorded a twofold increase in the prevalence of NAFLD over a decade. ${ }^{23,24}$ In contrast, the reported prevalence of NAFLD in the United States was already around 30-40\% in 2004 and has not increased substantially thereafter ${ }^{14}$. The incidence of NAFLD is difficult to study because ultrasonography cannot reliably quantify liver fat. Nevertheless, according to a recent study from Hong Kong using paired ${ }^{1} \mathrm{H}-\mathrm{MRS}, 13.5 \%$ of community subjects developed NAFLD in 3-5 years. ${ }^{25}$ Asians and Pacific Islanders develop obesity-related complications at a lower body mass index (BMI) than Europeans and Americans because of the tendency to have central fat accumulation. As a result, around 15$20 \%$ of Asian patients with NAFLD do not have obesity according to Western definitions.

Data on the prevalence of advanced disease in the general Asia-Pacific and African populations are scarce. One population study in Hong Kong using transient elastography and serum markers of fibrosis suggests that around $4 \%$ of the patients with NAFLD in the community have advanced fibrosis or cirrhosis. ${ }^{26}$ In high risk groups, such as patients with type 2 diabetes, advanced fibrosis and cirrhosis can be found in $10-20 \%$ of patients.

\section{[H2] Children and Adolescents}

[H3] The United States and Europe. NAFLD is the most common liver disease in children and adolescents (Table 2). Similarly to adult patients, NAFLD is associated with obesity and metabolic syndrome ${ }^{27}$ and probably with elevated alanine aminotransferase (ALT) levels in blood and PNPLA3 polymorphisms ${ }^{28}$ ( Figure 1$)^{29}$. A recent analysis of the National Health and Examination Survey, including data from $>12,000$ 12-19 year-old Americans, showed that NAFLD prevalence (with NAFLD defined as $B M I \geq 85^{\text {th }}$ percentile and elevated ALT levels of $>22.1 \mathrm{U} / \mathrm{L}$ for girls and $>25.8 \mathrm{U} / \mathrm{L}$ for boys) more than doubled over the past two decades ${ }^{27}$. The associated risk factors identified in this study confirm previously identified factors such as age, increased BMI, male gender and MexicanAmerican race ${ }^{30}$. Modifiable factors were increased consumption of sugar-sweetened beverages and 
other lifestyle factors (such as a sedentary behaviour) ${ }^{27}$. Paediatric NAFLD prevalence has been predicted to increase even further ${ }^{31}$.

Ethnicity has a role is NAFLD risk in children as well. Given similar BMI and metabolic syndrome features, black and non-Hispanic children have a lower risk of developing liver disease than Hispanic children (reviewed $\mathrm{in}^{29}$ ). Hereditability, particularly in first-degree relatives, is documented. Indeed, $59 \%$ of siblings and $78 \%$ of parents of children with fatty liver will develop NAFLD as well ${ }^{32}$. Age is another striking factor; one post-mortem study in California showed that $17 \%$ of teenagers had NAFLD compared to $0.7 \%$ of $2-4$ year olds, owing both to a longer period to accumulate steatosis as well as an increased incidence in adolescents ${ }^{30}$. [Au: OK? Ok now]

[H3] Asia, Africa and Pacific Islands. Given that obesity and overweight are important risk factors for NAFLD, the prevalence of NAFLD in Korea is greater in patients admitted to obesity clinics $(71.9 \%$ based on ultrasonography measures and $61.8 \%$ based on serum ALT levels) than in general health clinics ( $8.7 \%$ based on ultrasonography measures and $5.9 \%$ based on serum ALT levels) $)^{33}$. In most of Asia, a significant difference is reported between urban and rural populations with a prevalence of $16-32 \%$ in urban areas versus $9 \%$ in rural populations ${ }^{34}$. Obesity-related NAFLD was reported in 64 of 84 Chinese children (77\%) in one study ${ }^{35}$. In Australia, the prevalence of NAFLD in the paediatric population was estimated to be approximately $10 \%$ in the total population and $27.6 \%$ among overweight and obese children ${ }^{36}$.

\section{[H1] Mechanisms/pathophysiology}

\section{[H2] Pathophysiology of NAFLD}

Fatty liver disease is characterized by hepatic steatosis; there may also be varying degrees of liver cell injury, inflammation and in some cases, fibrosis (Figure 2).

[H3] The evolution of hepatic steatosis in obesity. Several factors contribute to hepatic lipid accumulation in the setting of obesity. First, weight gain is associated with marked expansion of adipose tissue, which leads to dysfunction and eventual death of adipocytes. Adipose tissue dysfunction results in local inflammation and up-regulation of cytokines that promote insulin resistance. Insulin-resistance, in turn, compromises the ability of adipocytes to store fat, resulting in release of free fatty acids into the circulation, which then become available for uptake by ectopic organs such as the liver. ${ }^{37}$ As a consequence of obesity-related adipose tissue dysfunction, the liver is 
exposed to high levels of circulating free fatty acids as well as high levels of insulin produced to compensate for adipocyte insulin resistance. Hepatocytes take up these fatty acids via transporters FATP5 and CD36, which are also up-regulated in obesity. ${ }^{38,39}$ Fatty acid accumulation in hepatocytes prompts the synthesis of triglyceride; during this process, diacylglycerol intermediates accumulate, which impair hepatic insulin signalling by activating protein kinase $C$ epsilon (PKC $\varepsilon$ ). ${ }^{40}$ Hepatocyte insulin resistance fuels gluconeogenesis, promoting hyperglycaemia and prompting even more compensatory insulin production.

The liver itself can contribute to steatosis by producing lipid from carbohydrate in a process called de novo lipogenesis (DNL). The enzymes responsible for DNL are up-regulated by insulin and glucose through the action of two transcription factors, sterol regulatory element binding protein-1 (SREBP1) and carbohydrate response element binding protein (ChREBP). ${ }^{41}$ In the normal liver, DNL is not a major source of hepatic lipid, but in the setting of obesity and hyperinsulinaemia, it can contribute as much as $25 \%$ of total hepatic lipid stores, and is considered an important factor in the development of NAFLD. ${ }^{42,43}$ Why an insulin-dependent process such as DNL would be up-regulated in hepatocytes if uptake of excess circulating fatty acids has rendered them insulin-resistant seems paradoxical. One explanation is that hepatocyte insulin resistance manifests downstream from the insulin receptor per se; ${ }^{44}$ another is that DNL can also be induced by insulin-independent pathways such as endoplasmic reticulum stress-mediated activation of SREBP $1^{45}$.

Nutrients from the diet also serve as substrates for hepatic triglyceride synthesis and thus participate in the development of hepatic steatosis. Dietary sugars are converted to fatty acids via DNL and dietary fats are taken up by the liver in parallel with adipose tissue-derived fatty acids. Roughly $40 \%$ of the lipid that accumulates in a fatty liver derives from dietary sugars and fats. Lipolysis of dysfunctional adipose tissue contributes the remaining $60 \%{ }^{46}$

[H3] The gut-liver axis in NAFLD. Interactions between the intestine and the liver are emerging as important determinants of NAFLD. Obesity, which represents the background for NAFLD, is associated with alterations in the intestinal microbiome that enhance nutrient extraction from the $\operatorname{diet}^{47}$. To date, NAFLD has not yet been linked to any specific microbiotic signature, but people with NAFLD have an intestinal microbiome that is clearly different from that of healthy individuals ${ }^{48}$. Intestinal dysbiosis can contribute to liver disease by weakening the intestinal barrier and enabling the translocation of bacteria or bacterial products such as endotoxin or ethanol into the portal 
circulation. Dysbiosis can also lead to the luminal degradation of beneficial nutrients such as choline, which are important for maintenance of hepatic lipid homeostasis ${ }^{11}$.

[H3] Mechanisms of cell death in a fatty liver. One of the hallmarks that differentiates NASH from NAFLD is the presence of hepatocellular injury. Hepatocytes can be damaged by several mechanisms in the setting of NAFLD. First, the demand for metabolism of excess fatty acids places strain on hepatocyte mitochondria; over time this leads to mitochondrial uncoupling, production of reactive oxygen species (ROS) and activation of Jun N-terminal kinase (JNK) ${ }^{49}$. Once initiated, ROS production and JNK activation continue in a feed-forward loop, which results in mitochondrial damage, impaired ATP production and cell death ${ }^{50,51}$. Excess fatty acids can also injure hepatocytes by inducing endoplasmic reticulum stress, which this leads to mitochondrial dysfunction and cell death via caspase-2-mediated cleavage of the BH3-only protein $\mathrm{BID}^{52-54}$. Yet another pathway via which fatty acids can kill hepatocytes is through activation of death receptors. Several death receptors (FAS, DR5 (also known as TRAIL-R2), TNFR1) are up-regulated on hepatocytes in the setting of steatosis; death receptor activation has been implicated as an important stimulus to hepatocyte apoptosis and necroptosis in $\mathrm{NASH}^{55-58}$.

[H3] Inflammation and fibrosis in fatty livers. Activation of the immune system is a key feature of $\mathrm{NASH}$. The classic effectors of hepatic inflammation in NASH are Kupffer cells and recruited macrophages, but natural killer T cells play an important part in macrophage recruitment ${ }^{59}$ and both natural killer $T$ and $T$ cells are emerging as contributors to progressive liver disease ${ }^{60-64}$. Several compounds can trigger inflammation in fatty livers - including fatty acids, bacterial endotoxin reaching the liver from the intestine, and damage-associated molecular patterns (DAMPs) released from dying hepatocytes - and induce inflammation by activating Toll-like receptors (TLRs) and inflammasomes in target immune cells ${ }^{65,66}$, stimulating the production of an array of cytokines and chemokines $^{59,67,68}$.

The development of hepatic fibrosis portends a poor outcome in NASH. ${ }^{7}$ The central event in hepatic fibrogenesis is activation of hepatic stellate cells ${ }^{69}$; these cells are susceptible to stimulation by a variety of compounds present in a diseased fatty liver. Like inflammatory cells, stellate cells can be activated by DAMPs from dying hepatocytes or other sources that engage TLRs on the cell surface ${ }^{70}$. Other compounds such as free cholesterol can amplify hepatic fibrosis by up-regulating TLR expression on stellate cells ${ }^{71}$. Oxidant stress can also activate stellate cells; advanced glycation end products, which are abundant in diabetics, induce ROS production by stellate cells by inducing the 
enzyme NOX2 $2^{72}$. One unique consideration in NASH is the role of ballooned hepatocytes in hepatic fibrosis. Ballooned cells produce sonic hedgehog ${ }^{73}$, a protein that promotes tissue fibrosis ${ }^{74}$. Whether hedgehog stimulates fibrosis through direct effects on stellate cells or indirect effects on fatty liver injury is currently under study ${ }^{75}$.

\section{[H2] Genetics}

A substantial proportion of the population are at risk of progressive NAFLD (that is, progression to NASH with advanced fibrosis/cirrhosis) owing to obesity and insulin resistance. However, only a minority of people with NAFLD progress to more advanced disease including hepatocellular carcinoma $^{76}$. The reasons for this remain incompletely understood, but NAFLD is best considered a complex disease trait, the development and progression of which are attributed to subtle interpatient variations including host genetic factors and environment interact to produce disease phenotype and determine disease progression.. Although the presence of NAFLD is principally determined by environmental factors, genetic factors contribute and, crucially, determine how individuals respond to the challenge of caloric excess and consequent metabolic stressors.

Until 2008 , studies looking for genetic variants involved in susceptibility to NAFLD have been restricted to case-control allelic association studies limited by the fact that they are reliant on $a$ priori hypotheses for gene selection. Candidate genes are drawn from the limited pool of genes of which biological function is understood and considered relevant to the disease. Only a minority of genes associated with NAFLD through candidate-gene analysis have been independently validated in large independent studies or through the use of transmission disequilibrium testing. This short list includes mitochondrial superoxide dismutase $2(S O D 2)^{77}$, phosphatidylethanolamine $\mathrm{N}$ methyltransferase $(P E M T)^{78}$, fatty acid desaturase $1^{79}$ and kruppel-like factor-6 $(K L F 6)^{80}$. All of these genes were associated with progressive NAFLD rather than NAFLD per se. More recently, a number of novel genetic associations with NAFLD have been identified through genome-wide association studies (GWAS) ${ }^{81-88}$. Two of these associations are worthy of particular attention due to their reproducibility in several GWASs, their association with histologically advanced disease in subsequent association studies and their biological plausibility - at least as genes clearly involved hepatic lipid metabolism.

The first of these genes encodes PNPLA ${ }^{81}$. The index single nucleotide polymorphism (SNP) in PNPLA3 (rs738409 c.444 C>G, p.I148M) is a non-synonymous cytosine to guanine nucleotide 
transversion mutation that results in an isoleucine to methionine amino acid change at codon 148 . A gene dosage effect for PNPLA3 ${ }^{148 M}$ carriage is observed with a stepwise increase in ${ }^{1} \mathrm{H}-\mathrm{MRS}$ determined hepatic triglyceride content (HTGC) with increasing carriage of the minor allele ${ }^{81}$. The minor-allele frequency also correlates with ethnic differences in susceptibility to NAFLD. Multiple histologically based studies have clearly demonstrated that the PNPLA3 ${ }^{1148 M}$ variant is associated with the severity of steatohepatitis and fibrosis ${ }^{89}$ and more recently with the presence of $\mathrm{HCC}^{90}$. PNPLA3 might, therefore, be used as part of an HCC surveillance strategy. The PNPLA3 ${ }^{1148 M}$ SNP has also been associated with a greater response to dietary or lifestyle modification and may therefore be a biomarker for both greater risk of progressive disease and also greater benefit from intervention. The precise mechanism by which the PNPLA3 ${ }^{148 M}$ SNP contributes to disease remains controversial despite intensive study, although alterations in lipid droplet architecture and in retinol metabolism of the hepatic stellate cell have been associated with the PNPLA3 ${ }^{1148 M}$ variant $^{91,92}$.

The second of these genes encodes a transmembrane superfamily member TM6SF2 on chromosome 19, with a non-synonymous SNP (rs58542926 c.449 C>T, p.E167K) associated with ${ }^{1} \mathrm{H}-\mathrm{MRS}$-quantified $\mathrm{HTGC}^{87}$. As with PNPLA3, candidate gene studies have shown that the TM6SF2 ${ }^{E 167 \mathrm{~K}}$ variant is also associated with progressive NAFLD ${ }^{93}$. Interestingly, evidence has been provided that carriage of the more common $T M 6 S F 2^{E 167 K}$ major allele is associated with dyslipidaemia (raised serum LDL cholesterol and triglyceride), increased myocardial infarction and cardiovascular disease risk whereas minor allele carriage is protective ${ }^{94}$. Functional studies suggest that TM6SF2 regulates hepatic lipid efflux, with its deletion or mutation resulting in a reduction in lipoprotein secretion (very low density lipoprotein (VLDL), triglycerides and APOB) leading to increased hepatocellular lipid droplet size and triglyceride accumulation. The $T M G S F 2^{E 167 K}$ results in lower total cholesterol in humans and expression levels of the variant are lower than the wild-type form. These data indicate that the TM6SF2 ${ }^{E 167 K}$ variant confers a 'loss-of-function' to the protein that may be both qualitative and quantitative ${ }^{94}$.

Although these studies fail to provide any explanation for the association between the TM6SF2 variant and progressive NAFLD they do suggest an interesting paradox - the 'TM6SF2 Catch-22' paradigm $^{95}$. Here, on a background of insulin resistance and metabolic stress, TM6SF2 genotype acts as a determinant of metabolic syndrome-related end-organ damage and clinical outcome: protecting the liver at the expense of increased risk of atherosclerosis and cardiovascular disease or vice versa. The best evidence in support of this paradigm has recently been provided by a study in patients with NAFLD demonstrating that although the minor threonine SNP $\left(T M 6 S F 2^{E 167 K}\right.$ ) is associated with 
advanced disease, carriage of the major cysteine allele $\left(T M 6 S F 2^{167 E}\right)$ is associated with dyslipidaemia and cardiovascular disease ${ }^{96}$.

\section{[H2] Epigenetics}

The role of epigenetic factors in the biology of NAFLD is starting to emerge and has enabled integrating the information on gene expression with the external and internal metabolic information (Figure 3). Epigenetic alterations orchestrate the reprogramming of transcriptional machinery of hepatocytes, which occurs during their adaptation to a lipotoxic environment, inflammation and oxidative stress. These epigenetic changes could at least partially explain why the degree of histological damage and/or recovery differs among individuals with an identical genetic background (for example presence of PNPLA3 ${ }^{148 M}$ risk allele ${ }^{97}$ ) and in response to similar injury (that is, fat overload) .

[H3] DNA methylation. DNA methylation in particular is suggested to be involved in the progression of NAFLD towards advanced (fibrotic) clinical stages. Joined global expression and methylation analysis of human liver specimens demonstrated that advanced disease is associated with global deregulation (mostly hypomethylation) of methylation in multiple $\mathrm{CpG}$ sites $^{98}$. Hypomethylation and increased expression of genes involved in tissue repair, regeneration and tumorigenesis were found, as were hypermethylated transcriptionally repressed genes that are involved in basic metabolic functions (such as lipid, urea and amino acid metabolism) or belonged to the cytochrome P450 family ${ }^{98}$. In addition, DNA hypomethylation was associated with transdifferentiation of stellate cells into myofibroblasts in cell models, a phenomenon commonly associated with fibrosis ${ }^{99}$. Abnormal methylation of the PNPLA3 promoter was associated with fibrosis severity in a large number of Japanese people ${ }^{100}$. Differences in the methylation status at specific $\mathrm{CpG}$ islands within the promoter of anti-fibrogenic (PPARA and PPARD) and pro-fibrogenic (TGFB1, COL1A1 and PDGFA) genes might have a prognostic value in differentiating patients with mild fibrosis from those with advanced fibrosis ${ }^{101}$.

Liver-specific epigenetic changes that affect physiological processes and lead to perturbations of normal hepatocyte and mitochondrial function not only affect the progression of NAFLD but also NAFLD-related metabolic phenotypes, such as insulin resistance. Targeted analysis of the methylation status of the peroxisome proliferator-activated receptor gamma coactivator $1 \alpha$ (PPARGC1A) - a master transcriptional regulator of glucose and lipid metabolism and mitochondrial biogenesis - showed that methylation levels significantly correlated with plasma insulin levels, $\beta$ cell function and the HOMA-IR marker for insulin resistance (calculated as (glucose $\mathrm{mmol} / \mathrm{L} \times$ insulin 
$\mathrm{mU} / \mathrm{ml} / 22.5$ ) in humans ${ }^{102}$. A complex interaction between PPARGC1A mRNA levels and liver mitochondrial DNA (mtDNA) copy number was also reported. Interestingly, low mtDNA copy number was also directly associated with insulin resistance and NAFLD ${ }^{102}$. metylation of mtDNA might have a role in modulating the liver phenotype beyond genomic DNA ${ }^{103}$. Specifically, methylation and transcriptional activation of the mitochondrial-encoded NADH dehydrogenase 6 (MT-ND6), which is involved in oxidative phosphorylation, in the liver was associated with more advanced liver pathology in humans ${ }^{103}$.

A possible explanation for the changes in DNA methylation in NAFLD is its association with obesity, which has been suggested to be associated with reduced DNA demethylation ${ }^{104}$. Furthermore, evidence suggests that the activity of 5-hydroxymethylcytosine $(5 \mathrm{hmc})$ might be an additional mechanism that explains liver DNA-demethylation pathways in patients with NAFLD ${ }^{105}$.

Epigenetic changes can be potentially inherited from one generation to the next. For instance, an experimental study in rodents showed that liver injury induced by the hepatotoxin carbon tetrachloride $\left(\mathrm{CCl}_{4}\right)$ led to hypomethylation in the PPARG gene promoter and associated chromatin modifications in sperm, in stem cells and/or mature sperm ${ }^{106}$. Although this experimental study suggests that the hepatic wound healing response might be heritable, it remains unknown whether the same is true in patients with NAFLD. In addition, epigenetic alterations acquired during development may contribute to foetal metabolic programming ${ }^{107}$. Maternal protein restriction in rodents led not only to fatty liver in the offspring but reduced life span; further in vitro analysis showed that these changes were associated with changes in DNA methylation ${ }^{108}$. Likewise, in utero overnutrition affects a sirtuin-mediated histone acetylase/deacetylase pathway that impacts on the hepatic histone code of the progeny, as shown in a nonhuman primate experimental model of high fat diet-induced NAFLD ${ }^{109}$.

[H3] Non-coding RNAs. A large part ( 60\%) of the cellular gene-expression machinery is regulated by non-coding RNAs (ncRNA) - RNA transcripts that do not encode proteins (Figure 3). MicroRNAs (miRNAs) have been studied extensively in liver disease research, and evidence for their role in NAFLD is consistent. A number of miRNAs have been implicated in NAFLD severity, liver injury and lipid metabolism, including miR-122, miR-192, mir-375 and miR-34a ${ }^{110-112}$.

Of particular importance in the pathophysiology of NAFLD is miR-122, expression of which is highly enriched in healthy liver. Its network of regulated-genes are involved in cholesterol metabolism ${ }^{113}$, 
modulation of liver inflammation and progression of hepatocarcinogenesis ${ }^{114}$. An in vitro study showed that miR-122 regulates collagen production in hepatic stellate cells suggesting a pivotal role in liver fibrogenesis ${ }^{115}$. Notably, expression levels of miR-122 are decreased in the liver of patients with NAFLD whereas levels are increased in the circulating (extracellular) compartment ${ }^{112}$; rodent models of diet-induced steatohepatitis confirmed these findings ${ }^{116}$. This marked down-regulation of liver miR-122 observed in NAFLD seems to be of particular relevance in the pathogenesis of the disease. In fact, silencing of miR-122 is an early event during hepatocarcinogenesis as a consequence of NASH in humans ${ }^{117}$. Whether the down-regulation of miR-122 in the liver is the cause or the consequence of the progression of NAFLD remains to be investigated, because of the cross-sectional nature of the above-mentioned studies.

Several studies have explored the role of circulating (extracellular) miR-122 levels as biomarker of NASH - or disease progression - in humans, because miR-122 levels significantly correlate with histological damage (including hepatocellular ballooning, degeneration and fibrosis) and elevated liver enzymes (a marker for liver inflammation or injury) ${ }^{112}$. A two-stage study, exploring a large panel of circulating miRNAs at different stages of NAFLD, showed that circulating levels of miR-122 were increased 7.2-fold in patients with NASH versus healthy controls, and 3.1-fold in NASH versus simple steatosis ${ }^{112}$. Although the performance of circulating miR-122 suggests that it could be a reliable biomarker of liver injury in NAFLD, the overall predictive value for liver fibrosis is very similar to currently available non-invasive measurements, including serum cytokeratin-18 levels ${ }^{112}$. Nevertheless, the research agenda is still open; for instance, preliminary data from a rat model of Roux-en-Y gastric bypass showed that circulating miR-122 levels decrease after surgery, suggesting a putative role as biomarker of response to therapeutic interventions ${ }^{118}$. Clinical evidence has suggested that bariatric surgery was able to partially reverse epigenetic alterations in the liver associated with NAFLD. Notably, reversing differentially methylated sites in NAFLD correlates with improvement in liver histology ${ }^{119}$.

Furthermore, miR-122 released from the liver circulate in the bloodstream - such as an hormone capable of integrating global metabolic signalling ${ }^{112}$. A functional polymorphism in the 3 ' UTR region of a validated miR-122 target gene (that is, rs41318021 of SLC7A1), was significantly associated with arterial hypertension, suggesting that miR-122 might potentially modulate the susceptibility to elevated blood pressure in people who carry the risk allele of this variant ${ }^{112}$. Moreover, the expression levels of liver miR-122 significantly and negatively correlated with systolic 
arterial blood pressure ${ }^{112}$. Together, the reduction of miR-122 levels in NAFLD might explain in part the connection between NAFLD and cardiovascular risk.

Finally, although the role of long non-coding RNAs (IncRNAs) in the pathophysiology of NAFLD remains largely unknown, that they are implicated in lipid homeostasis. For instance, a liver-enriched IncRNA in mouse regulates lipoprotein lipase activation through an FXR-mediated pathway ${ }^{120}$.

\section{[H1] Diagnosis, screening and prevention}

\section{[H2] Diagnosis of NAFLD}

Most patients with NAFLD are asymptomatic or complain about non-specific symptoms, such as fatigue, sleep disturbances or right upper quadrant discomfort. Hepatomegaly is the most common physical finding. More advanced liver disease is associated with signs of portal hypertension. Features of polycystic ovary syndrome (associated with hyperandrogenism) should be sought in young women with suspected NAFLD ${ }^{121,122}$. Obstructive sleep apnoea syndrome and psoriasis can be associated with NAFLD ${ }^{123}$. Apolipoprotein B and lysosomal acid lipase (LAL) partial deficiency are rare familial causes of NAFLD and should be suspected in the absence of a clear association with components of metabolic syndrome, although LAL partial deficiency is usually associated with high levels of total and LDL cholesterol ${ }^{124,125}$. Because no specific marker is currently available, the diagnosis of NAFLD (Figure 4) requires the exclusion of all the known other causes of chronic liver disease and of secondary causes of steatosis such as medications (Box 1). The former include, among others, heavy alcohol consumption, viral hepatitis (especially hepatitis C genotype 3 ), Wilson disease, haemochromatosis, autoimmune hepatitis. To discriminate NAFLD from AFLD, a threshold for daily alcohol intake has been set at <30 g for men and $<20 \mathrm{~g}$ for women ${ }^{126}$ but a clear differentiation between the two is difficult in those patients who consume a slightly higher amount of alcohol (up to $40 \mathrm{~g} /$ day in both men and women).

Another important criteria is that NAFLD is tightly associated with metabolic syndrome and insulin resistance in the liver, muscle and adipose tissues ${ }^{127}$. NAFLD should be suspected in all individuals with one or more components of the metabolic syndrome, defined as the cluster any three of the following five features: increased waist circumference (ethnicity adjusted), increased fasting glucose or type 2 diabetes, hypertriglyceridaemia, low high density lipoprotein (HDL) levels (gender adjusted) and elevated blood pressure ${ }^{128}$. This association does not characterize NAFLD due to genetic variant in PNPLA3, but PNPLA3 mutation and metabolic syndrome may be associated; that is, frequently present in the same person [Au:OK?] ${ }^{129}$. 
Diagnostic workup should include evaluation of family and personal history of components of the metabolic syndrome and assessment of liver tests, including platelets, albumin and coagulation, fasting blood glucose, triglycerides and HDL levels. The most common modes of presentation of NAFLD are detection of unexplained abnormal liver enzymes and/or of bright liver at ultrasonography.

[H3] Blood parameters. Liver tests can show mild (twofold to fivefold) elevations of alanine aminotransferase (ALT), alkaline phosphatase (ALP) and $\gamma$-glutamyltranspeptidase (GGT), but levels are normal in the majority of people with NAFLD $(80 \%)^{130}$. The ratio of ALT to aspartate aminotransferase (AST) is greater than 1 unless advanced fibrotic NAFLD is present or the patient has covert alcoholic fatty liver disease (AFLD). Increased GGT levels do not discriminate between AFLD and NAFLD, as raised GGT are commonly associated with metabolic disease ${ }^{131}$. Ferritin may be increased in up to $60 \%$ of patients, but is mainly a marker of subclinical inflammation, given that iron overload is uncommon in NAFLD $(4-6 \%)^{132}$. However, high ferritin levels (twofold to threefold) [Au:OK?] have been associated with more advanced disease ${ }^{133}$. Autoantibodies (ANA and SMA) are often present at low titres $(1 / 80,1 / 160)$ as compared to absent in healthy individuals.

A test of insulin sensitivity is mandatory in all suspected patients. The homeostatic model assessment of insulin resistance is a good surrogate for insulin resistance in non-diabetic subjects ${ }^{134}$, although there is no universal agreement on threshold defining the test abnormal. Diagnostic workup should eventually include an oral glucose tolerance test according to standard criteria ${ }^{135}$, which provides additional information about the glucose tolerance status.

[H3] Ultrasound-based techniques. The leading quantitative ultrasound biomarker for hepatic steatosis is the controlled attenuation parameter (CAP) ${ }^{136}$ determined, which is a quantitative measure of the attenuation (energy loss) of the ultrasound beam as it traverses the hepatic parenchyma. The rationale is that ultrasound energy is attenuated more by fat than by non-fatty tissue; hence, greater attenuation implies greater fat content. The parameter is measured with the same device used in vibration-controlled transient elastography (VCTE, see below) and reported in units of $\mathrm{dB} / \mathrm{m}^{137}$. An anatomic image is not acquired, therefore, the location where the measurements were made is not recorded. Numerous single-centre studies suggest that CAP can accurately identify patients with hepatic steatosis ${ }^{138-140}$. However, due to overlap in CAP values across steatosis grades, CAP has limited accuracy for steatosis grading ${ }^{138,139}$. Also, the reproducibility 
of CAP values in patients with NAFLD has not been studied extensively. Although CAP shows promise, more data is needed before CAP can be considered a valid biomarker of hepatic steatosis.

[H3] MRI. The leading quantitative magnetic resonance imaging (MRI) biomarker for hepatic steatosis is proton density fat fraction (PDFF $)^{141}$. This biomarker measures the relative proportion of mobile protons attributable to fat and correlates closely with biochemically determined hepatic triglyceride concentration ${ }^{141}$. [Au: Some information moved to the figure legend.] In numerous single-centre and multi-centre studies, PDFF has shown high accuracy for diagnosing, grading, and longitudinally monitoring steatosis in adults and children, including morbidly obese individuals ${ }^{142-147}$. Historically, measurement of PDFF required MRS, which usually is available only in academic centres and generally assesses only portions of the liver. In recent years, advanced MRI techniques have been developed that measure PDFF throughout the entire liver in a single breathhold, thereby eliminating sampling variability. These techniques are becoming commercially available on the latest generation of MR scanners made by the leading manufacturers. Importantly, PDFF measurements made on scanners of different field strength and manufacturer agree closely ${ }^{146-148}$. With appropriate quality control, these advanced PDFF-estimation techniques can be used in clinical trials (Figure 5A).

\section{[H2] Diagnosis of NASH}

The diagnosis of NAFLD can be derived from classic risk factor assessment along with biochemical measures, and ultrasonound and MR-based detection of hepatic steatosis, yet the most relevant challenge to the clinician is the distinction between simple fatty liver and NASH. NAFLD is rarely perceived by the patient as a health problem, but NASH may have an asymptomatic course to overt liver disease. The presence of normal liver enzymes in the majority (80\%) of subjects renders the identification of patients at risk particularly challenging ${ }^{130}$. Importantly, histological severity between patients with and those without abnormal liver aminotransferases does not differ.

The actual diagnosis of NASH requires microscopic evaluation of liver tissue, most commonly obtained by liver biopsy (Figure 6). Histological analysis shows the constellation of steatosis, hepatocyte ballooning and lobular inflammation, typically in zone 3 , the microcirculatory unit through which blood exits the liver ${ }^{149}$. Fibrosis better predicts outcomes and mortality ${ }^{5}$. Semiquantitative grading and staging systems have been developed (Box 2). These systems are primarily used to the assess the severity of lesions. Grading involves the assessment of the activity of the disease: steatosis, inflammation and hepatocyte ballooning, whereas staging involves the 
assessment of fibrosis location and parenchymal (vascular remodeling). Although grade might be reversible with appropriate intervention, stage is likely less reversible.

As liver disease progression has been associated with persistence or worsening of metabolic risk factors ${ }^{150}$, most predictive indices of disease severity are based on components of the metabolic syndrome along with biochemical and imaging indicators of advanced liver disease. However, the large number of patients with NAFLD and the risk of complications associated with liver biopsy have led to the refinement of non-invasive techniques to predict the histological features of NAFLD and NASH.

In clinical practice, interpretation of non-invasive markers should be performed by hepatologists according to the clinical context and considering all the other clinical and laboratory findings. Combining two unrelated markers is recommended, as no single test has an advantage over the others in the prediction of the severity of liver disease ${ }^{151}$. Among the different strategies, algorithms combining transient elastography (see below) and serum biomarkers are the most attractive and validated $^{151}$. However, in case of unexplained discordance of non-invasive tests, a liver biopsy should be performed. With these considerations in mind, a reasonable approach to NAFLD patient is to consider liver biopsy according to Figure 4 .

[H3] Biomarkers and scores. Screening of steatosis is usually based on ultrasonography in individual patients, but indices or biomarkers are preferred in larger-scale screening studies. The best validated biomarkers are the Fatty Liver Index (FLI), the Steatotest and the NAFLD liver fat score. Simple steatosis does not increase mortality but these biomarkers can variably predict metabolic and cardiovascular outcomes or mortality ${ }^{152}$. Serum markers of fibrosis seem to have a better performance, particularly the NAFLD Fibrosis score (NFS), FIB-4, BARD, and commercially available panels such as FibroTest, FibroMeter and the Enhanced Liver Fibrosis (ELF) test ${ }^{153}$ (Table 3). All of these tools have acceptable diagnostic accuracy, but only NFS and FIB-4 have been extensively validated $^{19}$. All these tests perform best at excluding severe fibrosis-cirrhosis, with negative predictive values $>90 \%$, but are typically less accurate in the determination of less severe fibrosis ${ }^{153}$. NFS, FIB-4, ELF and FibroTest can also predict overall, cardiovascular and liver-related mortality ${ }^{152}$. levels of cytokeratine-18 fragments, a marker of apoptosis, had a promising performance, but recent 
data have highlighted the limited accuracy ${ }^{154}$ and to date no recommendation can be issued for the non-invasive diagnosis of NASH.

[H3] Ultrasonography. Conventional ultrasonography, in which standard B-mode (grey scale) ultrasonographic images are acquired and interpreted qualitatively, is frequently used to screen for hepatic steatosis, but conventional ultrasonography is insensitive to mild steatosis, especially in obese individuals, and is limited by reader variability ${ }^{155,156}$.

[H3] Elastography. Imaging techniques collectively known as elastography have been developed to indirectly measure tissue stiffness non-invasively. The measurement involves the assessment of the propagation of shear waves within the liver, from which the hepatic stiffness is inferred. Depending on the device, results can be reported as shear wave speed in $\mathrm{m} / \mathrm{s}$ or as one of several elastic moduli (e.g., Young modulus, complex shear modulus) in $\mathrm{kPa}^{157}$. These various elastography-derived parameters have emerged as the leading imaging biomarkers of hepatic 'fibrosis'.

[H3] Ultrasound-based elastography. The first ultrasound-based method to measure liver stiffness was $\mathrm{VCTE}^{158}$. This technology uses a non-imaging device to track shear waves transmitted transiently into the liver by an external vibration source; an anatomical image is not acquired and the location from which the measurements are made is not recorded.

More recently, elastography capabilities have been added to clinical ultrasound scanners ${ }^{157}$. Elastography-enabled scanners use ultrasound to track shear waves generated transiently in situ within the liver by an ultrasound push pulse (also known as an acoustic radiation force impulse); measurement locations are recorded on an anatomical image $e^{157,159,160}$ (Figure 5b). Because each manufacturer uses proprietary hardware and software, measurements made with different devices and even with different transducers (probes) on the same device do not agree ${ }^{160,161}$. Thus, any given patient should be monitored with a single device and using the same probe. VCTE and elastographyenabled scanners have been shown to provide high accuracy for the diagnosis of advanced fibrosis in patients with viral hepatitis ${ }^{162,163}$. Single-centre studies suggest these devices accurately diagnose advanced fibrosis in NAFLD as well ${ }^{163}$, although multi-centre validation studies have not yet been performed. None of these devices can diagnose early fibrosis accurately. The technical success rate, reliability, robustness and precision of these devices for measuring stiffness parameters in NAFLD has not been extensively studied ${ }^{160}$. Until such data becomes available, caution is advised for use of these devices in NAFLD clinical trials. 
[H3] MR based elastography. Magnetic resonance (MR) elastography uses a specialized MRI sequence to visualize shear waves delivered continuously into the liver by an external acoustic source $^{164}$. The wave images are processed by a so-called inversion algorithm to generate 'elastograms' ${ }^{165}$ (Figure 5c). These computer-generated images depict the spatial distribution of a stiffness parameter known as the magnitude of the complex shear modulus (usually referred to as 'shear stiffness' in the medical literature). The hepatic stiffness is recorded from representative portions of the liver. MR elastography now is commercially available on commercial MRI scanners, with the same hardware to generate the shear waves and the same inversion algorithm to process the wave images. Small single-centre studies in healthy volunteers suggest that measurements made with different scanners agree ${ }^{166}$. Large studies in patients with NAFLD are needed to confirm crossplatform reproducibility.

In single-centre studies, MR elastography has been shown to diagnose advanced fibrosis in adult NAFLD with high accuracy ${ }^{167-169}$. Accuracy of MR elastography probably is higher than that of ultrasound-based elastography ${ }^{170}$. As with ultrasound-based techniques, accuracy for detection of early fibrosis is modest. The precision of MR elastography in the NAFLD population has not been studied extensively. Multi-centre validation studies are needed before MR elastography can replace biopsy in NAFLD clinical trials.

\section{[H2] Follow-up monitoring}

Patient monitoring should include routine biochemistry, non-invasive monitoring of fibrosis and the assessment of comorbidities. Given that the risk of developing type 2 diabetes and cardiovascular complications is increased in patients with NAFLD ${ }^{123}$, oral glucose tolerance tests should be performed whenever glucose metabolism is abnormal [Au:OK?] (that is, fasting glucose of 100-126 $\mathrm{mg} / \mathrm{dl} ; \mathrm{HbA} 1 \mathrm{c}$ of $5.7-6.4 \%)^{135}$ and carotid doppler ultrasonography [Au:OK? Or is this an ultrasound-based technique] $;{ }^{123}$ referral to other specialists might be required. The timing of follow-up testing is still debated, and a definite evaluation for risks of progression and costs associated with investigations has not been performed yet. In patients with simple fatty liver a reasonable approach is a follow up in primary care setting, unless worsening of metabolic risk factors occurs. In patients with NASH and/or fibrosis a yearly monitoring policy is advisable. If indicated, on a case-by-case basis a repeat biopsy could be performed after 5-7 years (Figure 4). 


\section{[H1] Management}

\section{[H2] Current management of NAFLD}

[Au: Are treatments (incl. lifestyle changes) only encouraged when NASH has developed (and not in the earlier stages - fatty liver, steatosis)? Please add a short sentence to specify this.]The first step in the management of NASH in most patients is implementation lifestyle modifications that focus on healthy eating habits and regular exercise ${ }^{171}$. Without biopsy confirmation of the presence of NASH, lifestyle modification is still appropriate to reduce cardiovascular risks in patients with NAFLD and also to treat NASH if it is present. Weight loss for overweight and obese patients is recommended based on the collective observations from multiple studies showing that histological improvement can occur when patients lose $5-10 \%$ of body weight ${ }^{172,173}$. Unfortunately, meaningful lifestyle modification is difficult to achieve and difficult to sustain for most people ${ }^{174,175}$ and, therefore, alternatives are needed to treat NASH and prevent the associated complications of the metabolic syndrome. At this time, no pharmaceutical treatments are approved for NASH, although many agents are in various stages of evaluation in clinical trials. Bariatric surgery is a good option for select obese patients with NASH as it typically leads to improvement in NASH ${ }^{176,177}$.

\section{[H2] Pathogenesis-based targets of therapy}

One perspective on the pathogenesis of NASH is that lipotoxic lipid species, which are derived from an excess supply of fatty acids in hepatocytes, mediate the cellular injury and resulting inflammatory and fibrotic response that results in the histological phenotype of $\mathrm{NASH}^{178-180}$. This paradigm enables us to predict therapeutic interventions that might prevent or treat NASH by targeting the oversupply of fatty acids to the liver, the generation of lipotoxic intermediates and the resulting dysfunctional cellular responses (Figure 7). Given that the best treatments of disease are those that target underlying causes at a fundamental level, an ideal approach would be to pharmacologically manipulate the pathways of energy metabolism responsible for the heterogeneity in human energy efficiency.

If the genomic or epigenetic changes responsible for these differences can be identified and exploited, then treatments could be effective for NASH as well as the underlying insulin resistance, hypertension, hyperlipidaemia and other manifestations of the metabolic syndrome that lead to disability and early death ${ }^{181}$. Animal studies have provided some insights into these differences but how these will be translated into treatment of human disease is yet to be shown. Examples include the divergence of rat strains into those having high and low exercise tolerance with features of the metabolic syndrome developing in the latter ${ }^{182}$, the role $\beta$-adrenergic stimulation of highly metabolic 
brown adipose tissue ${ }^{183}$, the role of serotonin in modulating metabolism ${ }^{184}$, and the role of peripheral cannabinoid signalling in modulating energy efficiency ${ }^{185}$. At this time, insights into the regulation of energy metabolism are mostly at early preclinical stages and clinical trials have generally focused on manipulating more downstream processes such as lipogenesis, lipolysis, inflammation, oxidant stress and fibrogenesis.

\section{[H2] Preventing an oversupply of fatty acids in the liver}

[H3] Inhibition of de novo lipogenesis. One approach to reducing lipotoxic liver injury is to reduce the endogenous production of fatty acids in the liver by inhibiting DNL. The transcription factor SREBP1C is a major regulator of DNL; thus, treatments that decrease SREBP1C may be beneficial. This is one potential mechanism by which the bile acid derivative obeticholic acid may work. As an activating ligand for the nuclear receptor FXR, obeticholic acid increases the expression of SHP1 which decreases the expression of SREBP1C ${ }^{186}$. A Phase II clinical trial of obeticholic acid has demonstrated histological improvement in NASH after 72 weeks (45\% versus $21 \%$ in placebo-treated patients, $P=0.0002)^{187}$. However, treatment was associated with significant pruritus in $23 \%$ in the treatment group compared with $6 \%$ in the placebo group and with a rise in total and LDL cholesterol and a decrease in HDL cholesterol in treated patients, the importance of which will be further assessed in another clinical trial.

Another approach to inhibiting DNL includes using inhibitors of key enzymes in the pathway of fatty acid synthesis such as acetyl-CoA carboxylase (ACC) and such agents are being evaluated in clinical trials. Augmenting the incretin axis with GLP-1 analogues such as exenatide or impairing the breakdown of endogenous GLP-1 with the DPP-4 inhibitors (gliptins) may also decrease hepatic lipogenesis by decreasing substrate availability and this approach is also being examined in clinical trials.

[H3] Reducing the burden of fatty acids delivered to the liver. Lipolysis of stored triglyceride in adipose tissue is the major source of fatty acids delivered via the circulation to the liver. Adipose tissue lipolysis is normally suppressed by insulin, but when adipose tissue is insulin resistant it continues to release fatty acids into the blood at inappropriate times contributing to the development of $\mathrm{NASH}^{188}$. The PPARy ligand thiazolidinediones (TZDs or 'glitazones') have been shown to improve adipocyte insulin responsiveness and thus decrease inappropriate lipolysis. One TZD, pioglitazone, has been shown to improve adipose insulin sensitivity and improve NASH ${ }^{189,190}$. Unfortunately, this benefit comes at the expense of increased body weight in some patients because 
of adipocyte triglyceride retention. This and possibly other adverse effects such as osteoporosis and bladder cancer have dampened the enthusiasm for using pioglitazone for NASH and it has not gained widespread use.

Metformin is another insulin sensitizing agent but its effect is primarily in the liver. There is little evidence that hepatic insulin resistance plays a role in causing NASH. A large randomized clinical trial (RCT) of metformin in children did not show any benefit on ALT levels or histology but fortunately neither did it show worsening ${ }^{191}$. Nonetheless, metformin may play an adjunctive role in the treatment of NASH because it is often associated with weight loss and recent data indicates that it is associated with a reduced risk of a number of malignancies ${ }^{192}$.

Fatty acids and the carbohydrates used to make fatty acids can also be diverted to oxidative pathways and this may explain the benefit of regular exercise in improving the metabolic syndrome. Oxidative pathways can be stimulated pharmacologically as well. The PPARA/PPARD ligand GFT505 was recently evaluated in a clinical trial because it improves insulin sensitivity, which may be explained by the effect of PPARA on promoting oxidative metabolism in the liver and PPARD in promoting oxidative metabolism in muscle ${ }^{193}$.

\section{[H2] Reducing injury}

The mechanisms of lipotoxic injury remain uncertain but oxidant stress has been proposed to have a role ${ }^{194}$. Oxidant stress with associated lipid peroxidation clearly occurs in $\mathrm{NASH}^{195}$, but whether it is causative or an epiphenomenon remains unknown. Two large RCTs of natural vitamin E (d- $\alpha$ tocopherol) at a dose of $800 \mathrm{IU}$ daily have been conducted, one in adults (PIVENS) and one in children (TONIC). The PIVENS trial demonstrated improvement in liver histology in $43 \%$ compared to a placebo response of $19 \%(P=0.001)^{190}$ and similarly the TONIC trial showed resolution of NASH in $58 \%$ compared to $28 \%$ in placebo $(P=0.006)^{191}$. For neither trial has there been evidence that indices of oxidant stress improved, so it remains somewhat unclear how vitamin E improves NASH. Both the PIVENS and TONIC trials were conducted in patients without diabetes or cirrhosis so the benefits of vitamin $\mathrm{E}$ in patients with these commonly associated conditions remain unknown. Concern has been raised about the cardiovascular safety of high dose vitamin E and, consequently, patients and doses should be carefully selected. The ideal patient may be someone with aggressive NASH and no diabetes or major risks for cardiovascular disease, but such patients comprise a relative minority. It should be noted that weight loss has an additive benefit in patients treated with vitamin $E$, so the use of vitamin $E$ should be an adjunct to weight loss and not an alternative ${ }^{196}$. Fish oil (poly $n-3$ fatty 
acids) may also exert beneficial effects on lipid peroxidation but a large trial of ethyl-eicosapentanoic acid in NASH patients did not show any benefit ${ }^{197}$.

Endoplasmic reticulum stress is another process known to occur in NASH that may be central to the pathogenesis of lipotoxic hepatocellular injury. Studies of agents that diminish the endoplasmic reticulum stress response have provided provocative data in animals but these have yet to be introduced into RCTs ${ }^{198}$.

The contribution of hypercholesterolaemia to the development of steatohepatitis and liver fibrosis has not been fully established but a number of studies suggest that it may have a role in some patients. A morphometric study of liver biopsies demonstrated cholesterol crystals in lipid droplets in patients with steatohepatitis but not steatosis ${ }^{199}$. Statin use is safe in patients with liver disease ${ }^{200}$ and a Cochrane review concluded that statins can improve steatosis and reduce aminotransferase levels but treatment trials with histological endpoints are lacking ${ }^{201}$. More recently, in a large European observational cohort with NAFLD, statin use was associated with less steatosis, NASH and fibrosis in a dose-dependent manner; interestingly the presence of the PNPLA3 ${ }^{1148 M}$ polymorphism prevented the protective effect of statins ${ }^{202}$. In another recent study, statin use in NAFLD patients was associated with a reduction in liver related deaths, liver transplants or other liver outcomes ${ }^{7}$.

\section{[H2] Inhibition of inflammation}

The role of inflammation in contributing to hepatocellular injury versus a consequence of injury is unknown but should be clarified by the results of clinical trials examining various anti-inflammatory approaches. One clinical trial of an anti-inflammatory phosphodiesterase-4 (PDE4) inhibitor was negative despite the demonstration of reduced circulating TNF $\alpha$ levels in patients given the active

agent ${ }^{203}$. However, like all contributors to the pathogenesis of NASH, inflammation may play an important role in only a subgroup of patients. Post-hoc analysis of RCT results to identify and then predict responders to a specific therapy will be essential for all agents.

\section{[H2] Inhibition of apoptosis}

Caspase inhibitors have been developed and are in RCTs for a number of diseases including NASH based on the recognized role of hepatocellular apoptosis in the pathogenesis of $\mathrm{NASH}^{204}$. Although this might be a rational approach in acute processes, theoretical concerns about the safety of long term inhibition of apoptosis persist with this treatment approach in NASH. 


\section{[H2] Inhibition of fibrosis}

In the absence of effective therapy for $\mathrm{NASH}$, being able to prevent the progression of fibrosis to cirrhosis would be advantageous. Even when effective NASH therapies are identified, there may be a role for short term use of effective antifibrotic agents to accelerate the reversal of fibrosis. No antifibrotic agents for liver disease are available but several are in RCTs including an anti-LOXL2 antibody and a galectin binding molecule that have shown early promise.

\section{[H1] Quality of life}

Quality of life (QOL) in patients with NAFLD is understudied, though its importance is substantial and broadens our understanding of the overall burden of the disease (Box 3). Five studies have reported QOL scores in the context of NAFLD ${ }^{205-207}$. Overall findings have been similar and suggest that NAFLD is associated with a reduced overall, but predominately physical health-related (HR)QOL. Some data suggest that the impairment in QOL reported in patients with NAFLD may be beyond that reported in other aetiologies of liver disease ${ }^{205,206}$. In a sample of 771 patients with biopsy-confirmed NAFLD from the NASH clinical research network database, QOL as measured by the short form (SF)-36 questionnaire, was compared to a United States reference population with or without chronic disease ${ }^{208}$. In this study, impaired QOL was most evident in physical health with mental health affected to a lesser degree.

Most patients with NAFLD are obese or overweight and even in those who are not, lifestyle change incorporating dietary modification and exercise are the cornerstone of therapy. Obesity has both health and psychosocial ramifications and can have a profound impact on an individual patient's QOL. In this cross-sectional study, obesity was not associated with lower QOL per se ${ }^{208}$; however, the effects of imposing lifestyle change were not measured. One study evaluated the impact on weight loss on HR-QOL in patients with NAFLD and found that compared to those who did not lose weight, those that did, had improved HR-QOL scores over baseline values, comparable to the general population ${ }^{207}$. A prospective study of intensive lifestyle intervention illustrated that patients who lost $>7 \%$ body weight benefitted not only from improvement or resolution in steatosis, necroinflammation and cellular ballooning (NASH resolution) but also fibrosis. ${ }^{173}$ Importantly, no patient who lost more than $7 \%$ body weight had progression of fibrosis. In those that lost $>10 \%$, fibrosis improved in $81 \%$, though overall numbers were small as was baseline extent of fibrosis. Weight loss and dietary change are hard to achieve and even harder to maintain, particularly in the 
case of morbid obesity. Not only is behavioural change difficult in itself, but barriers - physical, social and economic (amongst others) - can pose a challenge to accomplishing them effectively.

\section{[H2] Bariatric surgery for NASH}

Compared to obese patients matched for co-morbidities, those that undergo bariatric surgery have decreased long-term mortality ${ }^{209-211}$. The largest impact on mortality comes from a reduction in myocardial infarction and malignancy; the two most common causes of death in patients with NAFLD. Bariatric surgery can be an effective and appropriate treatment strategy for a subset of patients with NAFLD, including those with advanced fibrosis and even carefully selected patients with compensated cirrhosis who meet criteria for bariatric surgery. ${ }^{4,212}$. While it is important to note that bariatric surgery has not been studied prospectively specifically as a treatment for NASH, the best available data demonstrate that bariatric surgery in patients with NASH is safe and improves $\mathrm{NASH}$-related liver disease, including fibrosis ${ }^{177}$. The liver-related benefits derived from bariatric surgery such as roux-en-Y gastric bypass or sleeve gastrectomy, extend beyond weight loss. Both procedures increase levels of GLP-1, which decreases appetite, slows gastric emptying and improves insulin sensitivity. Furthermore, GLP-1 effects modulate bile acid signalling, specifically through the farsenoid $\mathrm{X}$ receptor (FXR) which can alter the gut microbiome and can have other potential benefits. ${ }^{213-215}$

However, practitioners and patients are reluctant to pursue bariatric surgery, even when patients are appropriate candidates. Although there are known complications that vary according to the procedure performed, the overall the risk is low in properly selected patients ${ }^{216}$. Although carefully selected patients with established compensated (stable without significant abnormalities in liver function) cirrhosis can safely undergo bariatric surgery, cirrhosis is associated with a higher risk of post-operative complications including hepatic decompensation (development of ascites, hepatic encephalopathy or variceal bleeding $)^{210}$. Careful patient selection can, therefore, not be overemphasized. Success of the intervention requires that patients remain committed to necessary lifestyle changes post-operatively to avoid regaining lost weight. More importantly, compliance with recommendations and follow-up are imperative to avoid metabolic and nutritional problems related to malabsorption or other consequences of bariatric surgery ${ }^{217}$.

\section{[H2] NASH and hepatocellular carcinoma}

Another consequence of liver disease in patients with NASH is HCC. The incidence of HCC is increasing both in the United States and worldwide. In Western countries, approximately $20 \%$ of 
HCCs are found in patients with NASH-related liver disease; and even more if one includes HCCs occurring in the setting of cryptogenic cirrhosis, which often represents 'burned out' NASH that has lost its classic histological features ${ }^{218,219}$. A recent study in the United States based on Surveillance, Epidemiology, and End Results (SEER)-Medicare registries from 2004-2009 found NASH to be the underlying cause of HCC in $14.1 \%$ of nearly 5,000 cases, following alcoholic liver disease in $16 \%$ and hepatitis $\mathrm{C}$ in $54.9 \%$. While this represented a $9 \%$ annual increase in NASH related HCC, NASH-HCC cases represented only $5 \%$ of liver transplants for HCC during the same time period, likely reflecting the burden of co-morbid illness in this population. Furthermore, patients with NASH and HCC were older and had shorter survival if they did not receive liver transplant compared to other aetiologies of liver disease $\mathrm{e}^{220}$

Wenzel and colleagues estimated the population attributable fraction (PAF), otherwise stated as the proportion of cases that can be attributed to specific risk factors, in patients with HCC from various causes of liver disease using the SEER-Medicare databases from 1994 to $2007^{221}$. Analysis of a cohort of 6,991 people showed that those with obesity and diabetes (presumably with NAFLD) had the highest PAF of HCC (36.6\%) compared with other aetiologies, in descending order of magnitude alcohol intake (23.5\%), hepatitis C (22.4\%), hepatitis B (6.3\%) and other (3.2\%). An important limitation of this study is that patients did not have a biopsy diagnosis of NAFLD or NASH. The PAF takes into account disease prevalence; thus, if NAFLD were to be eliminated, the impact on HCC would be substantial. However, the risk estimate of HCC in an individual patient with NAFLD is only in the order of 1.5-2.5 compared with 20.0-25.0 in a patient with hepatitis C. Per annum rates of HCC development in patients with NASH are lower than for patients with hepatitis C (2.6 vs. 4.0 $)^{21}$. Because NASH is so prevalent, it is not surprising that HCC as an indication for liver transplantation has increased 3.64 fold in patients with underlying NASH and a BMI>30 kg/m². In comparison, $\mathrm{HCC}$ due to hepatitis $C$ has increased only 2.25 fold over a 10 -year period from 2002-2012. While diabetes and obesity are independent risk factors for HCC, the increase in NASH related HCC persisted after controlling for these variables ${ }^{222}$. Although older age, obesity, type 2 diabetes mellitus and male sex are risk factors for the development of HCC, NASH does seem to contribute independently ${ }^{221,223}$.

Cirrhosis creates a milieu that is permissive for the development of HCC; thus, current screening recommendations focus primarily on cirrhotic patients. However, reports of HCC occurring in the absence of cirrhosis are on the rise, with some developing even in the absence of profound fibrosis. By some estimates, $10-75 \%$ of cases of HCC may occur in non-cirrhotic NAFLD 224,225 . This is a serious problem because of the large number of patients affected by NAFLD and NASH at a population level. The individual risk of HCC in non-cirrhotic patients with NAFLD is even less well understood. 
Consequently, although some individuals at risk for HCC may not be identified at an early stage of malignancy, there is presently insufficient evidence to alter current screening guidelines. Optimal recommendations for HCC screening in patients with NAFLD may change in the future as more is learned about individual risk in this challenging population.

\section{[H1] Outlook}

Both the short-term and long-term outlook for NAFLD will evolve as clinical and basic research continue to re-define the field (Box 4). NAFLD is an extremely complex and nuanced disease that represents the convergence of many pathways, risk factors and external influences that are not uniform in all patients. Therefore, it seems likely that NAFLD represents a constellation of various phenotypes that will require study from many angles as illustrated in this Primer. NAFLD is highly prevalent yet only a minor subset of patients progress to advanced liver disease, including HCC. Although many patients with NAFLD have a common metabolic profile (T2DM, hypertension, obesity amongst others) not all do. The underlying pathophysiology of NAFLD and in particular NASH is strongly linked to insulin resistance, aberrant hepatic lipid metabolism, visceral adiposity and inflammation. However several other important modulators of disease such as the environment and diet can further modify triggers of chronic extra- and intrahepatic immune pathways ${ }^{226}$, and the pathogenetic roles of gut dysbiosis ${ }^{227-229}$. The extent to which these factors drive disease progression and outcomes will be clarified as we gain further insight into the amassing data in this increasingly important area of research.

Furthermore, differences in genetic susceptibility as well as epigenetics may help to better understand at risk populations. Therefore, a more in-depth evaluation of these differences will allow us to better understand the phenotypes of NAFLD. In turn, a more sophisticated ability to phenotype patients with NAFLD will further clarify differences in disease progression between patients and permit a more personalized approach to therapy.

Animal models of disease have offered tremendous insight into relevant mechanisms, but to date, no animal model has faithfully replicated all aspects of the human condition. However this benchmark may not be compulsory or attainable given the heterogeneity of human disease.

Just as the pathophysiology of NAFLD is not the same in all patients, treatment will need to be individualized. With rare exception, a commitment to lifestyle modification should be the foundation of any treatment plan, but this is not sufficient for most patients. In the morbidly obese or those 
with severe co-morbid illness, bariatric surgery may have substantial benefit. Many patients will require more intense intervention - behavioural and pharmacological.

Until recently the landscape of clinical trials for NASH was somewhat limited. Currently there are hundreds of clinical trials in NASH. The breadth of mechanisms being targeted reflects the complexity of the disease. As the field continues to advance and patients can be better classified by constellations of risk factors, or sub-classification using genomics or metabolomics, we will be able to offer a more personalized, mechanistically-derived treatment approach to each patient. 


\section{Acknowledgements}

Elisabetta Bugianesi is a member of the EPoS (Elucidating Pathways of Steatohepatitis) consortium funded by the Horizon 2020 Framework Program of the European Union under Grant Agreement 634413.

\section{Author contributions}

Introduction (E.M.B.); Epidemiology (V.W.-S.W. and V.N.); Mechanisms/pathophysiology (C.P.D., S.S. and J.J.M); Diagnosis, screening and prevention (E.B. and C.B.S.); Management (B.A.N.-T.); Quality of life (M.E.R.); Outlook (M.E.R. [Au: OK?] ); overview of Primer (E.M.B.).

\section{Competing interests}

E.M.B has received consulting fees from Eli Lilly and acted as a paid study pathologist for Rottapharm. C.B.S. has received research grants from GE and Siemens. V.W.-S.W. is/has been an advisory board member for Gilead and Janssen, a consultant for AbbVie, Merck and NovoMedica; and has received lecture fees from AbbVie, Echosens and Gilead. B.A.N.-T. has received consulting fees from Nimbus Therapeutics, Bristol Myers Squibb, Janssen, Conatus, and Scholar. All other authors declare no conflict of interests. 
Box 1| Secondary causes of NAFLD

[H1] Drug use

- Tamoxifen

- Amiodarone

- Glucocorticoids

- Synthetic estrogens

- Antiviral agents (for example, highly active antiretroviral therapies)

- Methotrexate

[H1] Other metabolic or genetic causes

- Hypobetalipoproteinaemia

- Lysosomal acid lipase (LAL) partial deficiency

- Lipodystrophy

- Weber-Christian disease

[H1] Nutrition

- Malnutrition

- Malabsorption

- Total parenteral nutrition

- Rapid weight loss

- Jejunoileal bypass

[H1] Others

- Small bowel diverticulosis

- Exposure to petrochemicals

- Exposure to organic solvents 
Box 2 | Grading and staging histological features of NAFLD and NASH

[H1] NAFLD Activity Score (NAS from 0-8)*

- Steatosis ( $0<5 \% ; 1,5-33 \% ; 2,33-66 \%$; and 3, >66\%)

- Lobular inflammation, foci/20x magnification [Au:OK?] (0, not present; , 1=<2; 2, 2-4; and 3, $>4)$

- Ballooning (0, not present; 1, few; 2, prominent ballooning)

[H1] Fibrosis score (0-4)*

- 1a: Delicate zone 3 perisinusoidal fibrosis (psf) **

- $1 \mathrm{~b}$ : Dense zone 3 psf**

- 1c: Portal [Au:OK?] only

- 2: Zone $3+$ portal or periportal [Au:OK?]

- 3: Bridging (c-c, c-p, p-p)

- 4: Cirrhosis

[H1] Fatty Liver Algorithm***

- Steatosis (0-3)

- Activity (ballooning + lobular inflammation)

$\circ$ Ballooning is $0-2$

- Lobular Inflammation is $0-2$

- Fibrosis (similar to the fibrosis score of the NASH CRN above)

* NIDDK NASH CRN: Pathology Committee NAFLD Scoring System for Clinical Trials ${ }^{230}$. Although the NAS is known to closely correlate with a diagnosis of NASH, it was created for clinical trials in order to assess change in the components and has been shown to be associated with liver tests, (ALT and AST) while the pathologist's diagnosis associates with features of insulin resistance. The pathologist is encouraged to make a separate, pattern based diagnosis in addition to scoring the lesions

**Delicate psf requires trichrome stain, whereas dense psf can be visualized on H\&E stain first. ***A primary difference between the European algorithm and the CRN NAS is the former score was derived in order to equal a diagnosis. Steatosis $\geq 1$, activity $\geq 2$ (with both ballooning and lobular inflammation $\geq 1$ ) equals NASH. ${ }^{231}$ [Au: Reference here OK?] 
Box 3. Affected quality of life aspects in NAFLD

\section{[H1] Physical health scores}

- People with NASH have poorer scores than patients with NAFLD ${ }^{208}$

- NAFLD as an aetiology may have lower HR-QOL scores compared to other aetiologies of liver disease ${ }^{205,206}$

- Patients with cirrhosis have poorer scores than patients with no or any fibrosis ${ }^{205,206}$

- Weight loss in people with NAFLD may improve QOL scores ${ }^{207}$

[H1] Mental health scores ${ }^{205}$

- Not affected by degree of necroinflammatory injury

- Not affected by fibrosis stage or parenchymal remodelling (i.e. cirrhosis)

[H1] Bariatric surgery

- Improves overall survival in obesity ${ }^{173,207-210}$

- Safe in patients with NASH ${ }^{8}$ Improves necroinflammatory disease, steatosis and fibrosis 
Box 4. Clinical goals to diagnose and manage NAFLD

[H1] Diagnosis

- Validation of newer non-invasive detection methods to replace the use of invasive, expensive and imperfect 'gold standard' liver biopsy for detection and severity evaluation of NAFLD and NASH, including advanced fibrosis

- Serum-based algorithms as markers for liver injury and fibrosis ${ }^{19,232-236}$

- Imaging-based system to detect the constellation of lesions that collectively comprise NASH [Au:OK?]

\section{[H1] Management}

- Lifestyle intervention including management of meal content and portions and exercise programs

- Therapeutic treatments based on a better understanding of the mechanisms of liver injury from the underlying and closely related links of overweight, obesity and insulin resistance 
Figure 1: SNPs associated with NAFLD and NASH. [.];PNPLA3, adiponutrin; and TM6F2,

Figure 2. Mechanisms of steatosis and liver injury in NASH. (a) Hepatic fatty acids come from 3 sources: diet, de novo lipogenesis (DNL) and adipose tissue lipolysis. Under normal circumstances, most fatty acids come from adipose tissue lipolysis. In people with fatty livers, the absolute amount of fatty acids from all sources increases, but the proportion coming from DNL increases considerably. Long-chain saturated fatty acids, which are the products of DNL, are cytotoxic to liver cells. Conversion of these fatty acids to triglyceride (TG) limits their toxicity. TG is what is visible as steatosis. (b) Fatty acids can kill hepatocytes by up-regulating the expression of death receptors and their ligands, culminating in death receptor activation ('extrinsic' cell death). Fatty acids can also cause 'intrinsic' cell death by inducing ER stress leading to caspase-2-mediated cell death, in addition to JNK activation, production of reactive oxygen species (ROS) and mitochondrial dysfunction. In addition, fatty acids can stress mitochondria directly by creating high demand for beta-oxidation. This, too, can lead to excessive ROS production and mitochondrial dysfunction. Regardless of the pathway involved, fatty acid-induced cell death leads to the release of damageassociated molecular patterns (DAMPs) into the extracellular space. (c) Hepatic inflammation in fatty livers involves resident and recruited macrophages. These cells express TLRs and inflammasomes, which respond to DAMPs released from dead hepatocytes as well as other compounds in the local milieu such as fatty acids and bacterial endotoxin from the intestine. Activation of these pathways stimulates the production of pro-inflammatory cytokines. Hepatic fibrosis in NASH is prompted by the activation of hepatic stellate cells to collagen-producing myofibroblasts. These cells express TLRs, and as such are responsive to DAMPs and endotoxin; they are also stimulated by cytokines released by inflammatory cells. They produce cytokines as well as collagen, which can prompt a vicious cycle of inflammation and further fibrosis.

Figure 3: Overview of factors involved in the modulation of cellular epigenome.

The figure depicts overall mechanisms involved in the modulation of cellular epigenome These epigenetic changes, which are technically regarded as chemical modifications of genomic DNA, include DNA methylation with methylation leading to gene silencing and demethylation to transcriptional activation and post-translational modifications of histones, such as methylation and acetylation. Together, they lead to changes in the chromatin structure that ultimately influence gene expression. Epigenetic alterations can be either transient or permanent; the mentioned epigenetic mechanisms are quite dynamic and can be reverted by therapeutic or lifestyle interventional 
approaches. Epigenetic changes are shaped by both external and internal influences. For example, dietary components can influence activity of epigenetic modifications including histone acetyltransferases, histone deacetylases and histone and DNA methyltransferases. The cellular metabolic environment, including Intermediate metabolites and cofactors of the tricarboxylic acid (TCA) cycle, regulate the activity of enzymes involved in histone acetylation pathways and the balance between DNA methylation versus demethylation. Methylation of mtDNA by the DNA methyltransferase DNMT1 can contribute to the pathophysiology of complex diseases with mitochondrial dysfunction involvement, such as NAFLD.Finally, non-coding RNAs (including microRNAs (miRNAs) and long non-coding RNAs (LncRNAs)) can regulate transcription, methylation and chromatin structures

Figure 4. Proposed diagnostic flow of NAFLD and NASH. NAFLD should be suspected if patients show several aspects of metabolic syndrome or a bright liver on ultrasonography. After excluding secondary causes of steatosis, non-invasive test (biomarkers, serum parameters and imaging), before converting to the 'gold-standard', that is liver biopsy. However, this is and invasive procedure and is suboptimal for screening and frequent monitoring. HCC, hepatocellular carcinoma; LFT, liver tests; MetS, metabolic syndrome; US, ultrasound.

Figure 5: Diagnosis of liver fibrosis and NASH. [Au: Title added; OK?] A. Proton density fat fraction maps (PDFF), obtained by MRI, [Au:OK?] of an obese woman at multiple time points during a weight loss programme. PDFF is reported as a percentage, with a biological range in humans from $1 \%$ to $\sim 50 \%$. Depending on the reference standard, a PDFF cut-off value of 3-6\% differentiates nonsteatotic from steatotic liver. Notice progressive reduction in proton density fat fraction with reduction in body mass index (BMI). b-c| Same-day ultrasound-based shear wave elastography (b, US SWE) and MR elastography (c, MRE) in five adults with biopsy-proven NAFLD and fibrosis stages as shown. Notice that patients with greater fibrosis have greater US SWE-measured shear wave speed and greater MRE-measured stiffness (magnitude of complex shear modulus).

Figure 6: Histological aspects of NAFLD and NASH. A| Marked steatosis without inflammation, hepatocyte injury (ballooning) or fibrosis. The steatosis is concentrated in acinar zone 3 , the microcirculatory unit through which blood exits the liver around the terminal hepatic venule (in circle) and shows sparing of the periportal, zone 1 hepatocytes, the microcirculatory unit through 
which portal and systemic blood enter and mix. This is the adult pattern of NAFLD. B| A closer view of a terminal hepatic venule (asterix) with hepatocytes around it with steatosis (clear vacuoles) and ballooning (arrows). Ballooned hepatocytes are commonly larger than neighboring hepatocytes due to swelling; the cytoplasm appears to be flocculent. Mallory-Denk bodies may occur in NASH, but are smaller than in alcoholic steatohepatitis. The inflammation is largely comprised of mononuclear cells including lymphocytes and macrophages. $\mathrm{Cl}$ Perisinusoidal fibrosis in the perisinusoidal spaces of zone 3 is detected by trichrome staining, staining hepatocytes in red and fibrosis in blue.

Hepatocytes with steatosis (S), ballooned hepatocytes (B) and pigmented Kupffer cells (macrophages, arrow) are indicated. [Au:OK?] D | NASH with bridging fibrosis (trichrome staining, blue), which typically surrounds and nearly encases individual hepatocytes. e/ Cirrhotic NASH in which all lesions of active NASH remain present (steatosis (S), ballooning (B), inflammation (I), perisinuisoidal fibrosis (PSF)) $\mathrm{f} \mid \mathrm{NASH}$ with cirrhosis, but no active lesions remain. One would only know this was a case of NASH-related cirrhosis by having had a prior biopsy with the diagnosis of active NASH. g| Paediatric pattern of NAFLD. In this very low power view of liver, it is apparent that the fat vacuoles localize near the portal tracts, and not the terminal hepatic venules. One involved portal area is encircled; a spared zone 3 is in a box.

Figure 7. The lipotoxicity model of NASH and targets for therapy. Central to this understanding of the pathogenesis of NASH are free fatty acids, their sources in the hepatocyte and their fates. Delivery from adipose tissue and de novo lipogenesis (DNL) are the major sources of free fatty acids in hepatocytes. Mitochondrial beta-oxidation and secretion into the blood as triglyceride in very low density lipoprotein (VLDL) are the major fates. When the supply is excessive or the disposal is impaired (or most likely a combination of both), the generation of lipotoxic lipids can occur. This leads to hepatocellular injury, inflammation and the phenotype of NASH. These processes can be a stimulus for fibrogenesis and carcinogenesis. This paradigm predicts therapeutic targets (green dashed boxes) and targets (green), many of which are being exploited in clinical trials.

Table 1. Epidemiology of NAFLD in adults

\begin{tabular}{|l|l|l|l|l|}
\hline Study* & Country or region & Setting & $\begin{array}{l}\text { Prevalence } \\
\text { of NAFLD } \\
(\%)\end{array}$ & $\begin{array}{l}\text { Prevalence } \\
\text { of NASH (\%) }\end{array}$ \\
\hline
\end{tabular}




\begin{tabular}{|c|c|c|c|c|}
\hline West & & & & \\
\hline Autopsy $^{16}$ & Canada & $\begin{array}{l}\text { Hospital autopsy } \\
\text { record }\end{array}$ & $\begin{array}{l}\text { Non-obese:7 } \\
\text { Obese: } 29 * *\end{array}$ & $\begin{array}{l}\text { Mean: } 6 \\
\text { Non-obese: } \\
3 \\
\text { Obese: } 19 \\
* *\end{array}$ \\
\hline Histology ${ }^{17-19}$ & $\begin{array}{l}\text { United States, } \\
\text { Europe, Australia }\end{array}$ & $\begin{array}{l}\text { Hospital liver } \\
\text { biopsy series }\end{array}$ & $74-96$ & $6-55$ \\
\hline Ultrasonography ${ }^{10-13}$ & $\begin{array}{l}\text { United States, } \\
\text { Europe, Brazil }\end{array}$ & $\begin{array}{l}\text { General } \\
\text { population or } \\
\text { hospital clinics }\end{array}$ & $20-70$ & NR \\
\hline $\mathrm{CT}^{237}$ & United States & $\begin{array}{l}\text { General } \\
\text { population }\end{array}$ & 17 & NR \\
\hline $\begin{array}{l}\text { Proton-magnetic } \\
\text { resonance } \\
\text { spectroscopy }^{14}\end{array}$ & United States & $\begin{array}{l}\text { General } \\
\text { population }\end{array}$ & 34 & NR \\
\hline $\begin{array}{l}\text { Asia, Africa and Pacific } \\
\text { Islands }\end{array}$ & & & & \\
\hline Histology $^{25,110,238,239}$ & $\begin{array}{l}\text { Korea, Malaysia, } \\
\text { Hong Kong }\end{array}$ & $\begin{array}{l}\text { Liver donors and } \\
\text { hospital liver } \\
\text { biopsy series }\end{array}$ & $\begin{array}{l}\text { Liver donors: } \\
3 \\
\text { Liver biopsy } \\
\text { series: } 100\end{array}$ & $56-8 \%$ \\
\hline Ultrasonography ${ }^{240-243}$ & $\begin{array}{l}\text { Japan, China, Korea, } \\
\text { India }\end{array}$ & $\begin{array}{l}\text { General } \\
\text { population and } \\
\text { hospital health } \\
\text { checkup }\end{array}$ & $9-20$ & NR \\
\hline $\mathrm{CT}^{244,245}$ & Saudi Arabia, Egypt & $\begin{array}{l}\text { Hospital patients } \\
\text { and liver donors }\end{array}$ & $10-18$ & NR \\
\hline $\begin{array}{l}\text { Proton-magnetic } \\
\text { resonance } \\
\text { spectroscopy }^{25}\end{array}$ & Hong Kong & $\begin{array}{l}\text { General } \\
\text { population }\end{array}$ & 27 & NR \\
\hline $\begin{array}{l}* \text { When more than o } \\
\text { studies. }{ }^{*} \mathrm{BMI} \geq 30 \\
\text { steatohepatitis; } \mathrm{NR} \text {, nc }\end{array}$ & $\begin{array}{l}\text { ference is includ } \\
\text {; NAFLD, nonalco } \\
\text { orted. }\end{array}$ & $\begin{array}{l}\text { e data refer to } \\
\text { atty liver diseas }\end{array}$ & $\begin{array}{l}\mathrm{mmary} \text { of } \mathrm{s} \\
\mathrm{SH} \text {, nonalco }\end{array}$ & $\begin{array}{l}\text { eral } \\
\text { lic }\end{array}$ \\
\hline
\end{tabular}




\begin{tabular}{|c|c|c|c|c|}
\hline Study & Country & $n$ & $\begin{array}{l}\text { Prevalence of } \\
\text { NAFLD (\%) }\end{array}$ & $\begin{array}{l}\text { Prevalence of } \\
\text { NASH (\%) }\end{array}$ \\
\hline \multicolumn{5}{|l|}{ Elevated ALT } \\
\hline $\begin{array}{l}\text { Strauss et al. } \\
2000^{246}\end{array}$ & United States & 2,450 & 16 & $N / A$ \\
\hline Zou et al. $2005^{247}$ & China & 113 & $55.7^{*}$ & $\mathrm{~N} / \mathrm{A}$ \\
\hline \multicolumn{5}{|l|}{ Ultrasonography } \\
\hline $\begin{array}{l}\text { Guzzalonni et al. } \\
2000^{248}\end{array}$ & Italy & 375 & 38.7 & $\mathrm{~N} / \mathrm{A}$ \\
\hline Fu et al. $2006^{249}$ & China & 123 & 80.5 & 43.9 \\
\hline Chan et al. $2004^{35}$ & China & 84 & 77 & 24 \\
\hline \multicolumn{5}{|l|}{ Histology } \\
\hline $\begin{array}{l}\text { Schwimer et al. } \\
2003^{250}\end{array}$ & United States & 43 & $84^{*}$ & 63 \\
\hline $\begin{array}{l}\text { Nobili et al. } \\
2006^{251}\end{array}$ & Italy & 84 & $80 *$ & 26.2 \\
\hline $\begin{array}{l}\text { Feldstein et al. } \\
2009^{252}\end{array}$ & United States & 66 & 43 & 13.7 \\
\hline \multicolumn{5}{|l|}{ Autopsy } \\
\hline $\begin{array}{l}\text { Schwimmer et al. } \\
2006^{30}\end{array}$ & United States & 724 & 13 & 17.3 \\
\hline $\begin{array}{l}{ }^{*} \text { In overweight o } \\
\text { articles; ALT, alar }\end{array}$ & e populati & $A, n$ & t stated & erenced \\
\hline
\end{tabular}




\begin{tabular}{|c|c|c|c|c|c|c|c|c|}
\hline Score & Components & $\begin{array}{l}\text { Cut-off } \\
\text { value }\end{array}$ & AUROC & Sens $\%$ & $\begin{array}{l}\text { Spec } \\
\% \\
\end{array}$ & PPV & NPV & Notes \\
\hline \multicolumn{9}{|l|}{ NAFLD } \\
\hline $\begin{array}{l}\text { Fatty Liver } \\
\text { Index }(F L I)^{253}\end{array}$ & BMI, WC, TG, GGT & $>60$ & 0.85 & 61 & 86 & NA & NA & $\begin{array}{l}\text { Less } \\
\text { accurate in } \\
\text { Asian } \\
\text { population } \\
\text { Predicts } \\
\text { metabolic } \\
\text { and CV } \\
\text { outcomes, } \\
\text { and } \\
\text { hepatic } \\
\text { and CV } \\
\text { mortality. }\end{array}$ \\
\hline $\begin{array}{l}\text { NAFLD liver } \\
\text { fat score }\end{array}$ & MetS, T2DM, AST, ALT & -0.640 & 0.86 & 86 & 71 & NA & NA & $\begin{array}{l}\text { Prediction } \\
\text { not } \\
\text { improved } \\
\text { by PNPLA3 }\end{array}$ \\
\hline $\begin{array}{l}\text { SteatoTest }{ }^{\circledR} \\
255\end{array}$ & $\begin{array}{l}\text { GGT, ALT, BG, } \\
\text { TG, CHOL }\end{array}$ & $>0.69$ & 0.80 & 38 & 81 & 71 & 52 & $\begin{array}{l}\text { Predicts } \\
\text { overall } \\
\text { mortality }\end{array}$ \\
\hline \multicolumn{9}{|l|}{ Fibrosis } \\
\hline $\begin{array}{l}\text { NAFLD } \\
\text { fibrosis } \\
\text { score (NFS) } \\
256\end{array}$ & $\begin{array}{l}\text { Age, BG, BMI, } \\
\text { platelets, albumin, } \\
\text { AST/ALT }\end{array}$ & $\geq 0.676$ & 0.84 & 43 & 96 & 82 & 80 & $\begin{array}{l}\text { Predicts } \\
\text { liver- } \\
\text { related } \\
\text { events, } \\
\text { incident } \\
\text { diabetes, } \\
\text { all-cause } \\
\text { and CV } \\
\text { mortality }\end{array}$ \\
\hline $\begin{array}{l}\text { Fibro- } \\
\text { Test }^{\otimes^{257}}\end{array}$ & $\begin{array}{l}\text { GGT, BIL, } \\
\text { haptoglobin, } \\
\text { apoAl, } \\
\text { a2macroglobulin }\end{array}$ & $\begin{array}{l}>0.30 \\
>0.70\end{array}$ & 0.81 & $\begin{array}{l}92 \\
25\end{array}$ & $\begin{array}{l}71 \\
97\end{array}$ & $\begin{array}{l}33 \\
60\end{array}$ & $\begin{array}{l}92 \\
89\end{array}$ & $\begin{array}{l}\text { Predicts } \\
\text { overall } \\
\text { mortality, } \\
\text { progression } \\
\text { to } \\
\text { advanced } \\
\text { fibrosis; } \\
\text { associated } \\
\text { with CV } \\
\text { mortality }\end{array}$ \\
\hline $\begin{array}{l}\text { FIB-4 } \\
\text { index }\end{array}$ & $\begin{array}{l}\text { Age, ALT, AST, } \\
\text { platelets }\end{array}$ & $\geq 2.67$ & 0.80 & 33 & 98 & 80 & 83 & $\begin{array}{l}\text { Predicts all- } \\
\text { cause and } \\
\text { CV } \\
\text { mortality } \\
\text { and liver- } \\
\text { related } \\
\text { events }\end{array}$ \\
\hline BARD $^{235}$ & BMI, AST/ALT, & $2-4$ & 0.81 & NA & NA & 43 & 96 & $\begin{array}{l}\text { Predicts } \\
\text { liver- }\end{array}$ \\
\hline
\end{tabular}




\begin{tabular}{|c|c|c|c|c|c|c|c|c|}
\hline & T2DM & & & & & & & $\begin{array}{l}\text { related } \\
\text { events }\end{array}$ \\
\hline Hepascore ${ }^{259}$ & $\begin{array}{l}\text { Age, gender, } \\
\alpha 2 \text { macroglobulin, } \\
\text { HA, BIL, GGT }\end{array}$ & $>0.37$ & 0.81 & 75 & 84 & 57 & 92 & $\begin{array}{l}\text { Initially } \\
\text { developed } \\
\text { in } \\
\text { hepatitis C }\end{array}$ \\
\hline $\begin{array}{l}\text { Enhanced } \\
\text { Liver Fibrosis } \\
\text { (ELF) }\end{array}$ & $\begin{array}{l}\text { HA, TIMP1, } \\
\text { PIIINP }\end{array}$ & $>0.3576$ & 0.90 & 80 & 90 & 71 & 94 & $\begin{array}{l}\text { Predicts } \\
\text { overall and } \\
\text { CV } \\
\text { mortality }\end{array}$ \\
\hline $\begin{array}{l}\text { AST/platelet } \\
\text { ratio index } \\
\text { (APRI) }^{151}\end{array}$ & $\begin{array}{l}\text { AST, platelet } \\
\text { count }\end{array}$ & $\geq 0.918$ & 0.87 & 66 & 91 & 73 & 87 & $\begin{array}{l}\text { Predicts } \\
\text { liver- } \\
\text { related } \\
\text { events }\end{array}$ \\
\hline
\end{tabular}

Generally the performance of a non-invasive diagnostic method is evaluated by the area under the receiver operator characteristic curve (AUROC), taking liver biopsy as the reference standard, where a value of 1.0 reflects a 'perfect' test with $100 \%$ sensitivity (Sens) and specificity (Spec), and a value of 0.5 reflects a test as good as chance. PPV, positive predictive value; NPV, negative predictive value;, apoprotein Al; ALT, alanine aminotransferase; AST, aspartate aminotransferase; BG, fasting glucose; BIL, total bilirubin; BMI, body mass index; ALP, alkaline phosphatase; $\mathrm{CHOL}$, fasting cholesterol; CV, cardiovascular; GGT, $\gamma$-glutamyl-transpeptidase; HA, hyaluronic acid; HTN, hypertension; IR, insulin resistance; MetS, metabolic syndrome; NA, not available; PIIINP, aminoterminal propeptide of type III collagen; T2DM, type 2 diabetes mellitus; TG, fasting triglycerides; TIMP1, Tissue inhibitor of metalloproteinase 1; WC, waist circumference; NA, not available 
1. Yki-Järvinen, H. Non-alcoholic fatty liver disease as a cause and a consequence of metabolic syndrome. lancet. Diabetes Endocrinol. 2, 901-10 (2014).

2. Brunt, E. M. Non-alcoholic fatty liver disease: what's new under the microscope? Gut 60, 1152-8 (2011).

3. Torres, D. M. \& Harrison, S. A. Nonalcoholic fatty liver disease: Fibrosis portends a worse prognosis. Hepatology 61, 1462-4 (2015).

4. Adams, L. A. et al. The natural history of nonalcoholic fatty liver disease: a population-based cohort study. Gastroenterology 129, 113-21 (2005).

5. Ekstedt, M. et al. Fibrosis stage is the strongest predictor for disease-specific mortality in NAFLD after up to 33 years of follow-up. Hepatology 61, 1547-1554 (2015).

6. Baffy, G., Brunt, E. M. \& Caldwell, S. H. Hepatocellular carcinoma in non-alcoholic fatty liver disease: an emerging menace. J. Hepatol. 56, 1384-91 (2012).

7. Angulo, P. et al. Liver Fibrosis, but No Other Histologic Features, Is Associated With Long-term Outcomes of Patients With Nonalcoholic Fatty Liver Disease. Gastroenterology 149, 389397.e10 (2015).

8. Harmon, R. C., Tiniakos, D. G. \& Argo, C. K. Inflammation in nonalcoholic steatohepatitis. Expert Rev. Gastroenterol. Hepatol. 5, 189-200 (2011).

9. Rinella, M. E. Nonalcoholic Fatty Liver Disease. JAMA 313, 2263 (2015).

10. Araújo, L. M., De Oliveira, D. A. \& Nunes, D. S. Liver and biliary ultrasonography in diabetic and non-diabetic obese women. Diabetes Metab. 24, 458-62 (1998).

11. Bedogni, G. et al. Prevalence of and risk factors for nonalcoholic fatty liver disease: the Dionysos nutrition and liver study. Hepatology 42, 44-52 (2005).

12. Williams, C. D. et al. Prevalence of nonalcoholic fatty liver disease and nonalcoholic steatohepatitis among a largely middle-aged population utilizing ultrasound and liver biopsy: a prospective study. Gastroenterology 140, 124-31 (2011).

13. Zelber-Sagi, S., Nitzan-Kaluski, D., Halpern, Z. \& Oren, R. Prevalence of primary non-alcoholic fatty liver disease in a population-based study and its association with biochemical and anthropometric measures. Liver Int. 26, 856-63 (2006).

14. Browning, J. D. et al. Prevalence of hepatic steatosis in an urban population in the United States: impact of ethnicity. Hepatology 40, 1387-95 (2004).

15. Targher, G. et al. Prevalence of nonalcoholic fatty liver disease and its association with cardiovascular disease among type 2 diabetic patients. Diabetes Care 30, 1212-8 (2007).

16. Wanless, I. R. \& Lentz, J. S. Fatty liver hepatitis (steatohepatitis) and obesity: an autopsy study with analysis of risk factors. Hepatology 12, 1106-10 (1990). 
17. Silverman, J. F. et al. Liver pathology in morbidly obese patients with and without diabetes. Am. J. Gastroenterol. 85, 1349-55 (1990).

18. Matteoni, C. A. et al. Nonalcoholic fatty liver disease: a spectrum of clinical and pathological severity. Gastroenterology 116, 1413-9 (1999).

19. Dixon, J. B., Bhathal, P. S. \& O’Brien, P. E. Nonalcoholic fatty liver disease: predictors of nonalcoholic steatohepatitis and liver fibrosis in the severely obese. Gastroenterology 121, 91-100 (2001).

20. Hyysalo, J. et al. A population-based study on the prevalence of NASH using scores validated against liver histology. J. Hepatol. 60, 839-46 (2014).

21. Ascha, M. S. et al. The incidence and risk factors of hepatocellular carcinoma in patients with nonalcoholic steatohepatitis. Hepatology 51, 1972-8 (2010).

22. Wong, R. J. et al. Nonalcoholic steatohepatitis is the second leading etiology of liver disease among adults awaiting liver transplantation in the United States. Gastroenterology 148, 54755 (2015).

23. Fan, J.-G. Epidemiology of alcoholic and nonalcoholic fatty liver disease in China. J. Gastroenterol. Hepatol. 28 Suppl 1, 11-7 (2013).

24. Kojima, S.-I., Watanabe, N., Numata, M., Ogawa, T. \& Matsuzaki, S. Increase in the prevalence of fatty liver in Japan over the past 12 years: analysis of clinical background. J. Gastroenterol. 38, 954-61 (2003).

25. Wong, V. W.-S. et al. Incidence of non-alcoholic fatty liver disease in Hong Kong: a population study with paired proton-magnetic resonance spectroscopy. J. Hepatol. 62, 182-9 (2015).

26. Wong, V. W.-S. et al. Prevalence of non-alcoholic fatty liver disease and advanced fibrosis in Hong Kong Chinese: a population study using proton-magnetic resonance spectroscopy and transient elastography. Gut 61, 409-15 (2012).

27. Welsh, J. A., Karpen, S. \& Vos, M. B. Increasing prevalence of nonalcoholic fatty liver disease among United States adolescents, 1988-1994 to 2007-2010. J. Pediatr. 162, 496-500.e1 (2013).

28. Mangge, H. et al. Patatin-like phospholipase 3 (rs738409) gene polymorphism is associated with increased liver enzymes in obese adolescents and metabolic syndrome in all ages. Aliment. Pharmacol. Ther. 42, 99-105 (2015).

29. Nobili, V. et al. Nonalcoholic Fatty Liver Disease. JAMA Pediatr. 169, 170 (2015).

30. Schwimmer, J. B. et al. Prevalence of fatty liver in children and adolescents. Pediatrics 118, 1388-93 (2006).

31. Dunn, W. \& Schwimmer, J. B. The obesity epidemic and nonalcoholic fatty liver disease in children. Curr. Gastroenterol. Rep. 10, 67-72 (2008). 
32. Schwimmer, J. B. et al. Heritability of nonalcoholic fatty liver disease. Gastroenterology 136, 1585-92 (2009).

33. Yang, H. R., Yi, D. Y. \& Choi, H. S. Comparison between a pediatric health promotion center and a pediatric obesity clinic in detecting metabolic syndrome and non-alcoholic fatty liver disease in children. J. Korean Med. Sci. 29, 1672-7 (2014).

34. Loomba, R. \& Sanyal, A. J. The global NAFLD epidemic. Nat. Rev. Gastroenterol. Hepatol. 10, 686-90 (2013).

35. Chan, D. F. Y. et al. Hepatic steatosis in obese Chinese children. Int. J. Obes. Relat. Metab. Disord. 28, 1257-63 (2004).

36. Booth, M. L. et al. The population prevalence of adverse concentrations and associations with adiposity of liver tests among Australian adolescents. J. Paediatr. Child Health 44, 686-91 (2008).

37. Maher, J. J., Leon, P. \& Ryan, J. C. Beyond insulin resistance: Innate immunity in nonalcoholic steatohepatitis. Hepatology 48, 670-8 (2008).

38. He, J., Lee, J. H., Febbraio, M. \& Xie, W. The emerging roles of fatty acid translocase/CD36 and the aryl hydrocarbon receptor in fatty liver disease. Exp. Biol. Med. (Maywood). 236, 1116-21 (2011).

39. Mitsuyoshi, H. et al. Analysis of hepatic genes involved in the metabolism of fatty acids and iron in nonalcoholic fatty liver disease. Hepatol. Res. 39, 366-73 (2009).

40. Samuel, V. T. et al. Inhibition of protein kinase Cepsilon prevents hepatic insulin resistance in nonalcoholic fatty liver disease. J. Clin. Invest. 117, 739-45 (2007).

41. Postic, C. \& Girard, J. Contribution of de novo fatty acid synthesis to hepatic steatosis and insulin resistance: lessons from genetically engineered mice. J. Clin. Invest. 118, 829-38 (2008).

42. Lambert, J. E., Ramos-Roman, M. A., Browning, J. D. \& Parks, E. J. Increased de novo lipogenesis is a distinct characteristic of individuals with nonalcoholic fatty liver disease. Gastroenterology 146, 726-35 (2014).

43. Donnelly, K. L. et al. Sources of fatty acids stored in liver and secreted via lipoproteins in patients with nonalcoholic fatty liver disease. J. Clin. Invest. 115, 1343-51 (2005).

44. Li, S., Brown, M. S. \& Goldstein, J. L. Bifurcation of insulin signaling pathway in rat liver: mTORC1 required for stimulation of lipogenesis, but not inhibition of gluconeogenesis. Proc. Natl. Acad. Sci. U. S. A. 107, 3441-6 (2010).

45. Kammoun, H. L. et al. GRP78 expression inhibits insulin and ER stress-induced SREBP-1C activation and reduces hepatic steatosis in mice. J. Clin. Invest. 119, 1201-15 (2009).

46. Donnelly, K. L. et al. Sources of fatty acids stored in liver and secreted via lipoproteins in patients with nonalcoholic fatty liver disease. J. Clin. Invest. 115, 1343-51 (2005). 
47. $\mathrm{Wu}, \mathrm{G}$. D. et al. Linking long-term dietary patterns with gut microbial enterotypes. Science 334, 105-8 (2011).

48. Schnabl, B. \& Brenner, D. A. Interactions between the intestinal microbiome and liver diseases. Gastroenterology 146, 1513-24 (2014).

49. Koliaki, C. et al. Adaptation of hepatic mitochondrial function in humans with non-alcoholic fatty liver is lost in steatohepatitis. Cell Metab. 21, 739-46 (2015).

50. Begriche, K., Massart, J., Robin, M.-A., Bonnet, F. \& Fromenty, B. Mitochondrial adaptations and dysfunctions in nonalcoholic fatty liver disease. Hepatology 58, 1497-507 (2013).

51. Luedde, T., Kaplowitz, N. \& Schwabe, R. F. Cell death and cell death responses in liver disease: mechanisms and clinical relevance. Gastroenterology 147, 765-783.e4 (2014).

52. Upton, J.-P. et al. Caspase-2 cleavage of BID is a critical apoptotic signal downstream of endoplasmic reticulum stress. Mol. Cell. Biol. 28, 3943-51 (2008).

53. Johnson, E. S. et al. Metabolomic profiling reveals a role for caspase-2 in lipoapoptosis. J. Biol. Chem. 288, 14463-75 (2013).

54. Machado, M. V et al. Reduced lipoapoptosis, hedgehog pathway activation and fibrosis in caspase-2 deficient mice with non-alcoholic steatohepatitis. Gut 64, 1148-57 (2015).

55. Feldstein, A. E. et al. Hepatocyte apoptosis and fas expression are prominent features of human nonalcoholic steatohepatitis. Gastroenterology 125, 437-43 (2003).

56. Cazanave, S. C. et al. Death Receptor 5 Signaling Promotes Hepatocyte Lipoapoptosis. J. Biol. Chem. 286, 39336-39348 (2011).

57. Inokuchi-Shimizu, S. et al. TAK1-mediated autophagy and fatty acid oxidation prevent hepatosteatosis and tumorigenesis. J. Clin. Invest. 124, 3566-3578 (2014).

58. Gautheron, J. et al. A positive feedback loop between RIP3 and JNK controls non-alcoholic steatohepatitis. EMBO Mol. Med. 6, 1062-74 (2014).

59. Wehr, A. et al. Pharmacological inhibition of the chemokine CXCL16 diminishes liver macrophage infiltration and steatohepatitis in chronic hepatic injury. PLoS One 9, e112327 (2014).

60. Syn, W.-K. et al. Accumulation of natural killer T cells in progressive nonalcoholic fatty liver disease. Hepatology 51, 1998-2007 (2010).

61. Syn, W.-K. et al. NKT-associated hedgehog and osteopontin drive fibrogenesis in nonalcoholic fatty liver disease. Gut 61, 1323-1329 (2012).

62. Harley, I. T. W. et al. IL-17 signaling accelerates the progression of nonalcoholic fatty liver disease in mice. Hepatology 59, 1830-9 (2014).

63. Sutti, S. et al. Adaptive immune responses triggered by oxidative stress contribute to hepatic inflammation in NASH. Hepatology 59, 886-97 (2014). 
64. Wolf, M. J. et al. Metabolic activation of intrahepatic CD8+ T cells and NKT cells causes nonalcoholic steatohepatitis and liver cancer via cross-talk with hepatocytes. Cancer Cell 26, 549-64 (2014).

65. Roh, Y. S. \& Seki, E. Toll-like receptors in alcoholic liver disease, non-alcoholic steatohepatitis and carcinogenesis. J. Gastroenterol. Hepatol. 28 Suppl 1, 38-42 (2013).

66. Mehal, W. Z. The inflammasome in liver injury and non-alcoholic fatty liver disease. Dig. Dis. 32, 507-15 (2014).

67. Tosello-Trampont, A.-C., Landes, S. G., Nguyen, V., Novobrantseva, T. I. \& Hahn, Y. S. Kuppfer cells trigger nonalcoholic steatohepatitis development in diet-induced mouse model through tumor necrosis factor- $\alpha$ production. J. Biol. Chem. 287, 40161-72 (2012).

68. Leroux, A. et al. Toxic lipids stored by Kupffer cells correlates with their pro-inflammatory phenotype at an early stage of steatohepatitis. J. Hepatol. 57, 141-9 (2012).

69. Wallace, M. C., Friedman, S. L. \& Mann, D. A. Emerging and disease-specific mechanisms of hepatic stellate cell activation. Semin. Liver Dis. 35, 107-18 (2015).

70. Seki, E. et al. TLR4 enhances TGF-beta signaling and hepatic fibrosis. Nat. Med. 13, 1324-32 (2007).

71. Tomita, K. et al. Free cholesterol accumulation in hepatic stellate cells: Mechanism of liver fibrosis aggravation in nonalcoholic steatohepatitis in mice. Hepatology 59, 154-169 (2014).

72. Jiang, J. X. et al. Advanced glycation endproducts induce fibrogenic activity in nonalcoholic steatohepatitis by modulating TNF- $\alpha$-converting enzyme activity in mice. Hepatology $5 \mathbf{8}$, 1339-48 (2013).

73. Rangwala, F. et al. Increased production of sonic hedgehog by ballooned hepatocytes. J. Pathol. 224, 401-10 (2011).

74. Choi, S. S., Omenetti, A., Syn, W.-K. \& Diehl, A. M. The role of Hedgehog signaling in fibrogenic liver repair. Int. J. Biochem. Cell Biol. 43, 238-44 (2011).

75. Hirsova, P., Ibrahim, S. H., Bronk, S. F., Yagita, H. \& Gores, G. J. Vismodegib suppresses TRAILmediated liver injury in a mouse model of nonalcoholic steatohepatitis. PLoS One 8, e70599 (2013).

76. Anstee, Q. M., Targher, G. \& Day, C. P. Progression of NAFLD to diabetes mellitus, cardiovascular disease or cirrhosis. Nat. Rev. Gastroenterol. Hepatol. 10, 330-344 (2013).

77. Al-Serri, A. et al. The SOD2 C47T polymorphism influences NAFLD fibrosis severity: evidence from case-control and intra-familial allele association studies. J. Hepatol. 56, 448-54 (2012).

78. Dong, H. et al. The phosphatidylethanolamine N-methyltransferase gene V175M single nucleotide polymorphism confers the susceptibility to NASH in Japanese population. $J$. Hepatol. 46, 915-20 (2007). 
79. Wang, L. et al. Fatty acid desaturase 1 gene polymorphisms control human hepatic lipid composition. Hepatology 61, 119-28 (2015).

80. Miele, L. et al. The Kruppel-like factor 6 genotype is associated with fibrosis in nonalcoholic fatty liver disease. Gastroenterology 135, 282-291.e1 (2008).

81. Romeo, S. et al. Genetic variation in PNPLA3 confers susceptibility to nonalcoholic fatty liver disease. Nat. Genet. 40, 1461-5 (2008).

82. Chalasani, N. et al. Genome-wide association study identifies variants associated with histologic features of nonalcoholic Fatty liver disease. Gastroenterology 139, 1567-76, 1576.e1-6 (2010).

83. Speliotes, E. K. et al. Genome-wide association analysis identifies variants associated with nonalcoholic fatty liver disease that have distinct effects on metabolic traits. PLoS Genet. 7, e1001324 (2011).

84. Anstee, Q. \& Darlay, R. Genome-Wide Association Analysis Confirms Importance of PNPLA3 and Identifies Novel Variants Associated With Histologically Progressive. ... (2012). at $<$ https://scholar.google.co.uk/scholar?hl=en\&q=Genome-

Wide+Association+Analysis+Confirms+Importance+of+PNPLA3+and+Identifies+Novel+Variant s+Associated+With+Histologically+Progressive+Steatohepatitis+in+NAFLD\&btnG=\&as_sdt=1 \%2C5\&as_sdtp=\#0>

85. Kawaguchi, T. et al. Genetic polymorphisms of the human PNPLA3 gene are strongly associated with severity of non-alcoholic fatty liver disease in Japanese. PLoS One 7, e38322 (2012).

86. Feitosa, M. F. et al. The ERLIN1-CHUK-CWF19L1 gene cluster influences liver fat deposition and hepatic inflammation in the NHLBI Family Heart Study. Atherosclerosis 228, 175-80 (2013).

87. Kozlitina, J. et al. Exome-wide association study identifies a TM6SF2 variant that confers susceptibility to nonalcoholic fatty liver disease. Nat. Genet. 46, 352-6 (2014).

88. DiStefano, J. K. et al. Genome-wide analysis of hepatic lipid content in extreme obesity. Acta Diabetol. 52, 373-82 (2015).

89. Valenti, L. et al. Homozygosity for the patatin-like phospholipase-3/adiponutrin I148M polymorphism influences liver fibrosis in patients with nonalcoholic fatty liver disease. Hepatology 51, 1209-17 (2010).

90. Liu, Y.-L. et al. Carriage of the PNPLA3 rs738409 C >G polymorphism confers an increased risk of non-alcoholic fatty liver disease associated hepatocellular carcinoma. J. Hepatol. 61, 75-81 (2014).

91. Smagris, E. et al. Pnpla3I148M knockin mice accumulate PNPLA3 on lipid droplets and develop hepatic steatosis. Hepatology 61, 108-18 (2015).

92. Pirazzi, C. et al. PNPLA3 has retinyl-palmitate lipase activity in human hepatic stellate cells. Hum. Mol. Genet. 23, 4077-85 (2014). 
93. Liu, Y.-L. et al. TM6SF2 rs58542926 influences hepatic fibrosis progression in patients with non-alcoholic fatty liver disease. Nat. Commun. 5, 4309 (2014).

94. Holmen, O. L. et al. Systematic evaluation of coding variation identifies a candidate causal variant in TM6SF2 influencing total cholesterol and myocardial infarction risk. Nat. Genet. 46, 345-51 (2014).

95. Kahali, B. et al. TM6SF2: catch-22 in the fight against nonalcoholic fatty liver disease and cardiovascular disease? Gastroenterology 148, 679-84 (2015).

96. Dongiovanni, P. et al. Transmembrane 6 superfamily member 2 gene variant disentangles nonalcoholic steatohepatitis from cardiovascular disease. Hepatology 61, 506-14 (2015).

97. Sookoian, S. \& Pirola, C. J. Meta-analysis of the influence of I148M variant of patatin-like phospholipase domain containing 3 gene (PNPLA3) on the susceptibility and histological severity of nonalcoholic fatty liver disease. Hepatology 53, 1883-94 (2011).

98. Murphy, S. K. et al. Relationship between methylome and transcriptome in patients with nonalcoholic fatty liver disease. Gastroenterology 145, 1076-87 (2013).

99. Mann, J. et al. Regulation of myofibroblast transdifferentiation by DNA methylation and MeCP2: implications for wound healing and fibrogenesis. Cell Death Differ. 14, 275-85 (2007).

100. Kitamoto, T. et al. Targeted-bisulfite sequence analysis of the methylation of $\mathrm{CpG}$ islands in genes encoding PNPLA3, SAMM50, and PARVB of patients with non-alcoholic fatty liver disease. J. Hepatol. 63, 494-502 (2015).

101. Zeybel, M. et al. Differential DNA methylation of genes involved in fibrosis progression in non-alcoholic fatty liver disease and alcoholic liver disease. Clin. Epigenetics 7, 25 (2015).

102. Sookoian, S. et al. Epigenetic regulation of insulin resistance in nonalcoholic fatty liver disease: impact of liver methylation of the peroxisome proliferator-activated receptor $\gamma$ coactivator $1 \alpha$ promoter. Hepatology 52, 1992-2000 (2010).

103. Pirola, C. J. et al. Epigenetic modification of liver mitochondrial DNA is associated with histological severity of nonalcoholic fatty liver disease. Gut 62, 1356-63 (2013).

104. Horvath, S. et al. Obesity accelerates epigenetic aging of human liver. Proc. Natl. Acad. Sci. U. S. A. 111, 15538-43 (2014).

105. Pirola, C. J. et al. Epigenetic Modifications in the Biology of Nonalcoholic Fatty Liver Disease: The Role of DNA Hydroxymethylation and TET Proteins. Medicine (Baltimore). 94, e1480 (2015).

106. Zeybel, M. et al. Multigenerational epigenetic adaptation of the hepatic wound-healing response. Nat. Med. 18, 1369-77 (2012).

107. Sookoian, S., Gianotti, T. F., Burgueño, A. L. \& Pirola, C. J. Fetal metabolic programming and epigenetic modifications: a systems biology approach. Pediatr. Res. 73, 531-42 (2013). 
108. Carr, S. K. et al. Maternal diet amplifies the hepatic aging trajectory of Cidea in male mice and leads to the development of fatty liver. FASEB J. 28, 2191-201 (2014).

109. Suter, M. A. et al. A maternal high-fat diet modulates fetal SIRT1 histone and protein deacetylase activity in nonhuman primates. FASEB J. 26, 5106-14 (2012).

110. Shen, J. et al. Non-invasive diagnosis of non-alcoholic steatohepatitis by combined serum biomarkers. J. Hepatol. 56, 1363-70 (2012).

111. Min, H.-K. et al. Increased hepatic synthesis and dysregulation of cholesterol metabolism is associated with the severity of nonalcoholic fatty liver disease. Cell Metab. 15, 665-74 (2012).

112. Pirola, C. J. et al. Circulating microRNA signature in non-alcoholic fatty liver disease: from serum non-coding RNAs to liver histology and disease pathogenesis. Gut 64, 800-12 (2015).

113. Esau, C. et al. miR-122 regulation of lipid metabolism revealed by in vivo antisense targeting. Cell Metab. 3, 87-98 (2006).

114. Hsu, S.-H. et al. Essential metabolic, anti-inflammatory, and anti-tumorigenic functions of miR-122 in liver. J. Clin. Invest. 122, 2871-83 (2012).

115. Li, J. et al. miR-122 regulates collagen production via targeting hepatic stellate cells and suppressing P4HA1 expression. J. Hepatol. 58, 522-8 (2013).

116. Csak, T. et al. microRNA-122 regulates hypoxia-inducible factor-1 and vimentin in hepatocytes and correlates with fibrosis in diet-induced steatohepatitis. Liver Int. 35, 532-41 (2015).

117. Takaki, Y. et al. Silencing of microRNA-122 is an early event during hepatocarcinogenesis from non-alcoholic steatohepatitis. Cancer Sci. 105, 1254-60 (2014).

118. Wu, Q. et al. Metabolic phenotype-microRNA data fusion analysis of the systemic consequences of Roux-en-Y gastric bypass surgery. Int. J. Obes. (Lond). 39, 1126-34 (2015).

119. Ahrens, M. et al. DNA methylation analysis in nonalcoholic fatty liver disease suggests distinct disease-specific and remodeling signatures after bariatric surgery. Cell Metab. 18, 296-302 (2013).

120. Li, P. et al. A liver-enriched long non-coding RNA, IncLSTR, regulates systemic lipid metabolism in mice. Cell Metab. 21, 455-67 (2015).

121. Vassilatou, E. Nonalcoholic fatty liver disease and polycystic ovary syndrome. World J. Gastroenterol. 20, 8351-63 (2014).

122. Hossain, N. et al. Non-alcoholic steatohepatitis (NASH) in patients with polycystic ovarian syndrome (PCOS). Scand. J. Gastroenterol. 46, 479-84 (2011).

123. Armstrong, M. J., Adams, L. A., Canbay, A. \& Syn, W.-K. Extrahepatic complications of nonalcoholic fatty liver disease. Hepatology 59, 1174-97 (2014). 
124. Reiner, Ž. et al. Lysosomal acid lipase deficiency--an under-recognized cause of dyslipidaemia and liver dysfunction. Atherosclerosis 235, 21-30 (2014).

125. Bernstein, D. L., Hülkova, H., Bialer, M. G. \& Desnick, R. J. Cholesteryl ester storage disease: review of the findings in 135 reported patients with an underdiagnosed disease. J. Hepatol. 58, 1230-43 (2013).

126. Ratziu, V., Bellentani, S., Cortez-Pinto, H., Day, C. \& Marchesini, G. A position statement on NAFLD/NASH based on the EASL 2009 special conference. J. Hepatol. 53, 372-84 (2010).

127. Bugianesi, E., McCullough, A. J. \& Marchesini, G. Insulin resistance: a metabolic pathway to chronic liver disease. Hepatology 42, 987-1000 (2005).

128. Alberti, K. G. M. M., Zimmet, P. \& Shaw, J. The metabolic syndrome--a new worldwide definition. Lancet 366, 1059-62

129. Romeo, S. et al. Genetic variation in PNPLA3 confers susceptibility to nonalcoholic fatty liver disease. Nat. Genet. 40, 1461-5 (2008).

130. Browning, J. D. Statins and hepatic steatosis: perspectives from the Dallas Heart Study. Hepatology 44, 466-71 (2006).

131. Fraser, A. et al. Gamma-glutamyltransferase is associated with incident vascular events independently of alcohol intake: analysis of the British Women's Heart and Health Study and Meta-Analysis. Arterioscler. Thromb. Vasc. Biol. 27, 2729-35 (2007).

132. Bugianesi, E. et al. Relative contribution of iron burden, HFE mutations, and insulin resistance to fibrosis in nonalcoholic fatty liver. Hepatology 39, 179-87 (2004).

133. Ratziu, V., Bellentani, S., Cortez-Pinto, H., Day, C. \& Marchesini, G. A position statement on NAFLD/NASH based on the EASL 2009 special conference. J. Hepatol. 53, 372-84 (2010).

134. Matthews, D. R. et al. Homeostasis model assessment: insulin resistance and beta-cell function from fasting plasma glucose and insulin concentrations in man. Diabetologia 28, 412-9 (1985).

135. Standards of medical care in diabetes--2014. Diabetes Care 37 Suppl 1, S14-80 (2014).

136. Sasso, M. et al. Controlled attenuation parameter (CAP): a novel VCTE ${ }^{\mathrm{TM}}$ guided ultrasonic attenuation measurement for the evaluation of hepatic steatosis: preliminary study and validation in a cohort of patients with chronic liver disease from various causes. Ultrasound Med. Biol. 36, 1825-35 (2010).

137. Sasso, M. et al. Controlled attenuation parameter (CAP): a novel $\mathrm{VCTE}^{\mathrm{TM}}$ guided ultrasonic attenuation measurement for the evaluation of hepatic steatosis: preliminary study and validation in a cohort of patients with chronic liver disease from various causes. Ultrasound Med. Biol. 36, 1825-35 (2010).

138. Chan, W.-K., Nik Mustapha, N. R. \& Mahadeva, S. Controlled attenuation parameter for the detection and quantification of hepatic steatosis in nonalcoholic fatty liver disease. $J$. Gastroenterol. Hepatol. 29, 1470-6 (2014). 
139. Myers, R. P. et al. Controlled Attenuation Parameter (CAP): a noninvasive method for the detection of hepatic steatosis based on transient elastography. Liver Int. 32, 902-10 (2012).

140. Karlas, T. et al. Non-invasive assessment of hepatic steatosis in patients with NAFLD using controlled attenuation parameter and 1H-MR spectroscopy. PLoS One 9, e91987 (2014).

141. Reeder, S. B., Hu, H. H. \& Sirlin, C. B. Proton density fat-fraction: a standardized MR-based biomarker of tissue fat concentration. J. Magn. Reson. Imaging 36, 1011-4 (2012).

142. Tang, A. et al. Accuracy of MR imaging-estimated proton density fat fraction for classification of dichotomized histologic steatosis grades in nonalcoholic fatty liver disease. Radiology 274, 416-25 (2015).

143. Tang, A. et al. Nonalcoholic fatty liver disease: MR imaging of liver proton density fat fraction to assess hepatic steatosis. Radiology 267, 422-31 (2013).

144. Schwimmer, J. B. et al. Magnetic resonance imaging and liver histology as biomarkers of hepatic steatosis in children with nonalcoholic fatty liver disease. Hepatology 61, 1887-95 (2015).

145. Permutt, Z. et al. Correlation between liver histology and novel magnetic resonance imaging in adult patients with non-alcoholic fatty liver disease - MRI accurately quantifies hepatic steatosis in NAFLD. Aliment. Pharmacol. Ther. 36, 22-9 (2012).

146. Artz, N. S. et al. Reproducibility of MR-based liver fat quantification across field strength: Same-day comparison between 1.5T and 3T in obese subjects. J. Magn. Reson. Imaging 42, 811-7 (2015).

147. Hines, C. D. G. et al. T1 independent, T2* corrected chemical shift based fat-water separation with multi-peak fat spectral modeling is an accurate and precise measure of hepatic steatosis. J. Magn. Reson. Imaging 33, 873-881 (2011).

148. Hansen, K. H., Schroeder, M. E., Hamilton, G., Sirlin, C. B. \& Bydder, M. Robustness of fat quantification using chemical shift imaging. Magn. Reson. Imaging 30, 151-7 (2012).

149. Kleiner, D. E. \& Brunt, E. M. Nonalcoholic fatty liver disease: pathologic patterns and biopsy evaluation in clinical research. Semin. Liver Dis. 32, 3-13 (2012).

150. Marchesini, G. et al. Nonalcoholic fatty liver, steatohepatitis, and the metabolic syndrome. Hepatology 37, 917-23 (2003).

151. EASL-ALEH Clinical Practice Guidelines: Non-invasive tests for evaluation of liver disease severity and prognosis. J. Hepatol. 63, 237-64 (2015).

152. Fedchuk, L. et al. Performance and limitations of steatosis biomarkers in patients with nonalcoholic fatty liver disease. Aliment. Pharmacol. Ther. 40, 1209-22 (2014).

153. Castera, L., Vilgrain, V. \& Angulo, P. Noninvasive evaluation of NAFLD. Nat. Rev. Gastroenterol. Hepatol. 10, 666-75 (2013). 
154. Kwok, R. et al. Systematic review with meta-analysis: non-invasive assessment of nonalcoholic fatty liver disease--the role of transient elastography and plasma cytokeratin-18 fragments. Aliment. Pharmacol. Ther. 39, 254-69 (2014).

155. Machado, M. V \& Cortez-Pinto, H. Non-invasive diagnosis of non-alcoholic fatty liver disease. A critical appraisal. J. Hepatol. 58, 1007-19 (2013).

156. Awai, H. I., Newton, K. P., Sirlin, C. B., Behling, C. \& Schwimmer, J. B. Evidence and recommendations for imaging liver fat in children, based on systematic review. Clin. Gastroenterol. Hepatol. 12, 765-73 (2014).

157. Tang, A., Cloutier, G., Szeverenyi, N. M. \& Sirlin, C. B. Ultrasound Elastography and MR Elastography for Assessing Liver Fibrosis: Part 1, Principles and Techniques. AJR. Am. J. Roentgenol. 205, 22-32 (2015).

158. Sandrin, L. et al. Transient elastography: a new noninvasive method for assessment of hepatic fibrosis. Ultrasound Med. Biol. 29, 1705-13 (2003).

159. Bamber, J. et al. EFSUMB guidelines and recommendations on the clinical use of ultrasound elastography. Part 1: Basic principles and technology. Ultraschall Med. 34, 169-84 (2013).

160. Ferraioli, G. et al. WFUMB guidelines and recommendations for clinical use of ultrasound elastography: Part 3: liver. Ultrasound Med. Biol. 41, 1161-79 (2015).

161. Myers, R. P. et al. Feasibility and diagnostic performance of the FibroScan XL probe for liver stiffness measurement in overweight and obese patients. Hepatology 55, 199-208 (2012).

162. Bota, S. et al. Meta-analysis: ARFI elastography versus transient elastography for the evaluation of liver fibrosis. Liver Int. 33, 1138-47 (2013).

163. Friedrich-Rust, M. et al. Acoustic radiation force impulse-imaging and transient elastography for non-invasive assessment of liver fibrosis and steatosis in NAFLD. Eur. J. Radiol. 81, e32531 (2012).

164. Muthupillai, R. et al. Magnetic resonance elastography by direct visualization of propagating acoustic strain waves. Science 269, 1854-7 (1995).

165. Venkatesh, S. K., Yin, M. \& Ehman, R. L. Magnetic resonance elastography of liver: technique, analysis, and clinical applications. J. Magn. Reson. Imaging 37, 544-55 (2013).

166. Serai, S. D., Yin, M., Wang, H., Ehman, R. L. \& Podberesky, D. J. Cross-vendor validation of liver magnetic resonance elastography. Abdom. Imaging 40, 789-794 (2014).

167. Kim, D., Kim, W. R., Talwalkar, J. A., Kim, H. J. \& Ehman, R. L. Advanced fibrosis in nonalcoholic fatty liver disease: noninvasive assessment with MR elastography. Radiology 268, 411-9 (2013).

168. Loomba, R. et al. Magnetic resonance elastography predicts advanced fibrosis in patients with nonalcoholic fatty liver disease: a prospective study. Hepatology 60, 1920-8 (2014). 
169. Arulanandan, A. et al. Association Between Quantity of Liver Fat and Cardiovascular Risk in Patients With Nonalcoholic Fatty Liver Disease Independent of Nonalcoholic Steatohepatitis. Clin. Gastroenterol. Hepatol. 13, 1513-1520.e1 (2015).

170. Ichikawa, S. et al. Comparison of the diagnostic accuracies of magnetic resonance elastography and transient elastography for hepatic fibrosis. Magn. Reson. Imaging 33, 26-30 (2015).

171. Neuschwander-Tetri, B. A. Lifestyle modification as the primary treatment of NASH. Clin. Liver Dis. 13, 649-65 (2009).

172. Johnson, N. A. \& George, J. Fitness versus fatness: moving beyond weight loss in nonalcoholic fatty liver disease. Hepatology 52, 370-81 (2010).

173. Vilar-Gomez, E. et al. Weight Loss via Lifestyle Modification Significantly Reduces Features of Nonalcoholic Steatohepatitis. Gastroenterology 149, 367-378.e5 (2015).

174. Bellentani, S., Dalle Grave, R., Suppini, A. \& Marchesini, G. Behavior therapy for nonalcoholic fatty liver disease: The need for a multidisciplinary approach. Hepatology 47, 746-54 (2008).

175. Centis, E., Marzocchi, R., Di Domizio, S., Ciaravella, M. F. \& Marchesini, G. The effect of lifestyle changes in non-alcoholic fatty liver disease. Dig. Dis. 28, 267-73 (2010).

176. Pillai, A. A. \& Rinella, M. E. Non-alcoholic fatty liver disease: is bariatric surgery the answer? Clin. Liver Dis. 13, 689-710 (2009).

177. Lassailly, G. et al. Bariatric Surgery Reduces Features of Nonalcoholic Steatohepatitis in Morbidly Obese Patients. Gastroenterology 149, 379-388 (2015).

178. Neuschwander-Tetri, B. A. Hepatic lipotoxicity and the pathogenesis of nonalcoholic steatohepatitis: The central role of nontriglyceride fatty acid metabolites. Hepatology 52, 774-788 (2010).

179. Cusi, K. Role of obesity and lipotoxicity in the development of nonalcoholic steatohepatitis: pathophysiology and clinical implications. Gastroenterology 142, 711-725.e6 (2012).

180. Fuchs, M. \& Sanyal, A. J. Lipotoxicity in NASH. J. Hepatol. 56, 291-3 (2012).

181. Bouret, S., Levin, B. E. \& Ozanne, S. E. Gene-environment interactions controlling energy and glucose homeostasis and the developmental origins of obesity. Physiol. Rev. 95, 47-82 (2015).

182. Wisløff, U. et al. Cardiovascular risk factors emerge after artificial selection for low aerobic capacity. Science 307, 418-20 (2005).

183. Cypess, A. M. et al. Activation of human brown adipose tissue by a $\beta 3$-adrenergic receptor agonist. Cell Metab. 21, 33-8 (2015).

184. Crane, J. D. et al. Inhibiting peripheral serotonin synthesis reduces obesity and metabolic dysfunction by promoting brown adipose tissue thermogenesis. Nat. Med. 21, 166-72 (2014). 
185. Neuschwander-Tetri, B. A. Food energy efficiency, cannabinoids, and a slow death of the weight loss dogma. Hepatology 46, 12-5 (2007).

186. Kalaany, N. Y. \& Mangelsdorf, D. J. LXRS AND FXR: The Yin and Yang of Cholesterol and Fat Metabolism. Annu. Rev. Physiol. 68, 159-191 (2006).

187. Neuschwander-Tetri, B. A. et al. Farnesoid X nuclear receptor ligand obeticholic acid for noncirrhotic, non-alcoholic steatohepatitis (FLINT): a multicentre, randomised, placebocontrolled trial. Lancet 385, 956-965 (2015).

188. Lomonaco, R. et al. Effect of adipose tissue insulin resistance on metabolic parameters and liver histology in obese patients with nonalcoholic fatty liver disease. Hepatology 55, 1389-97 (2012).

189. Gastaldelli, A. et al. Importance of changes in adipose tissue insulin resistance to histological response during thiazolidinedione treatment of patients with nonalcoholic steatohepatitis. Hepatology 50, 1087-93 (2009).

190. Sanyal, A. \& Chalasani, N. Pioglitazone, vitamin E, or placebo for nonalcoholic steatohepatitis. ... Engl. J. ... (2010). at <http://www.nejm.org/doi/full/10.1056/nejmoa0907929>

191. Lavine, J. E. et al. Effect of vitamin E or metformin for treatment of nonalcoholic fatty liver disease in children and adolescents: the TONIC randomized controlled trial. JAMA 305, 165968 (2011).

192. Doycheva, I. \& Loomba, R. Effect of metformin on ballooning degeneration in nonalcoholic steatohepatitis (NASH): when to use metformin in nonalcoholic fatty liver disease (NAFLD). Adv. Ther. 31, 30-43 (2014).

193. Cariou, B., Zaïr, Y., Staels, B. \& Bruckert, E. Effects of the new dual PPAR $\alpha / \delta$ agonist GFT505 on lipid and glucose homeostasis in abdominally obese patients with combined dyslipidemia or impaired glucose metabolism. Diabetes Care 34, 2008-14 (2011).

194. Rolo, A. P., Teodoro, J. S. \& Palmeira, C. M. Role of oxidative stress in the pathogenesis of nonalcoholic steatohepatitis. Free Radic. Biol. Med. 52, 59-69 (2012).

195. Musso, G. et al. Nitrosative stress predicts the presence and severity of nonalcoholic fatty liver at different stages of the development of insulin resistance and metabolic syndrome: possible role of vitamin A intake. Am. J. Clin. Nutr. 86, 661-71 (2007).

196. Hoofnagle, J. H. et al. Vitamin E and changes in serum alanine aminotransferase levels in patients with non-alcoholic steatohepatitis. Aliment. Pharmacol. Ther. 38, 134-43 (2013).

197. Sanyal, A. J., Abdelmalek, M. F., Suzuki, A., Cummings, O. W. \& Chojkier, M. No significant effects of ethyl-eicosapentanoic acid on histologic features of nonalcoholic steatohepatitis in a phase 2 trial. Gastroenterology 147, 377-84.e1 (2014).

198. Cao, S. S. \& Kaufman, R. J. Targeting endoplasmic reticulum stress in metabolic disease. Expert Opin. Ther. Targets 17, 437-48 (2013). 
199. Ioannou, G. N., Haigh, W. G., Thorning, D. \& Savard, C. Hepatic cholesterol crystals and crown-like structures distinguish NASH from simple steatosis. J. Lipid Res. 54, 1326-34 (2013).

200. Bays, H., Cohen, D. E., Chalasani, N., Harrison, S. A. \& The National Lipid Association's Statin Safety Task Force. An assessment by the Statin Liver Safety Task Force: 2014 update. J. Clin. Lipidol. 8, S47-57

201. Eslami, L., Merat, S., Malekzadeh, R., Nasseri-Moghaddam, S. \& Aramin, H. Statins for nonalcoholic fatty liver disease and non-alcoholic steatohepatitis. Cochrane database Syst. Rev. 12, CD008623 (2013).

202. Dongiovanni, P. et al. Statin use and non-alcoholic steatohepatitis in at risk individuals. J. Hepatol. 63, 705-12 (2015).

203. Ratziu, V. et al. Lack of efficacy of an inhibitor of PDE4 in phase 1 and 2 trials of patients with nonalcoholic steatohepatitis. Clin. Gastroenterol. Hepatol. 12, 1724-30.e5 (2014).

204. Ratziu, V. et al. A phase 2, randomized, double-blind, placebo-controlled study of GS-9450 in subjects with nonalcoholic steatohepatitis. Hepatology 55, 419-28 (2012).

205. Dan, A. A. et al. Health-related quality of life in patients with non-alcoholic fatty liver disease. Aliment. Pharmacol. Ther. 26, 815-20 (2007).

206. AFENDY, A. et al. Predictors of health-related quality of life in patients with chronic liver disease. Aliment. Pharmacol. Ther. 30, 469-476 (2009).

207. Hickman, I. J. et al. Modest weight loss and physical activity in overweight patients with chronic liver disease results in sustained improvements in alanine aminotransferase, fasting insulin, and quality of life. Gut 53, 413-9 (2004).

208. David, K. et al. Quality of life in adults with nonalcoholic fatty liver disease: baseline data from the nonalcoholic steatohepatitis clinical research network. Hepatology 49, 1904-12 (2009).

209. Bray, G. A. The Missing Link - Lose Weight, Live Longer. N. Engl. J. Med. 357, 818-820 (2007).

210. Sjöström, L. et al. Effects of bariatric surgery on mortality in Swedish obese subjects. N. Engl. J. Med. 357, 741-52 (2007).

211. Adams, T. D. et al. Long-term mortality after gastric bypass surgery. N. Engl. J. Med. 357, 75361 (2007).

212. Sjöström, L. et al. Lifestyle, diabetes, and cardiovascular risk factors 10 years after bariatric surgery. N. Engl. J. Med. 351, 2683-93 (2004).

213. Meek, C. L., Lewis, H. B., Reimann, F., Gribble, F. M. \& Park, A. J. The effect of bariatric surgery on gastrointestinal and pancreatic peptide hormones. Peptides (2015). at <http://www.ncbi.nlm.nih.gov/pubmed/26344355>

214. Ryan, K. K. et al. FXR is a molecular target for the effects of vertical sleeve gastrectomy. Nature 509, 183-8 (2014). 
215. Kuipers, F. \& Groen, A. K. FXR: the key to benefits in bariatric surgery? Nat. Med. 20, 337-8 (2014).

216. Birkmeyer, N. J. O. et al. Hospital complication rates with bariatric surgery in Michigan. JAMA 304, 435-42 (2010).

217. Jan, A., Narwaria, M. \& Mahawar, K. K. A Systematic Review of Bariatric Surgery in Patients with Liver Cirrhosis. Obes. Surg. 25, 1518-26 (2015).

218. Yang, J. D. et al. Hepatocellular carcinoma in olmsted county, Minnesota, 1976-2008. Mayo Clin. Proc. 87, 9-16 (2012).

219. Malik, S. M., Gupte, P. A., de Vera, M. E. \& Ahmad, J. Liver transplantation in patients with nonalcoholic steatohepatitis-related hepatocellular carcinoma. Clin. Gastroenterol. Hepatol. 7, 800-6 (2009).

220. Younossi, Z. M. et al. Association of Non-alcoholic Fatty Liver Disease (NAFLD) with Hepatocellular Carcinoma (HCC) in the United States from 2004-2009. Hepatology (2015). at <http://www.ncbi.nlm.nih.gov/pubmed/26274335>

221. Welzel, T. M. et al. Population-attributable fractions of risk factors for hepatocellular carcinoma in the United States. Am. J. Gastroenterol. 108, 1314-21 (2013).

222. Wong, R. J., Cheung, R. \& Ahmed, A. Nonalcoholic steatohepatitis is the most rapidly growing indication for liver transplantation in patients with hepatocellular carcinoma in the U.S. Hepatology 59, 2188-2195 (2014).

223. Wideroff, L. et al. Cancer incidence in a population-based cohort of patients hospitalized with diabetes mellitus in Denmark. J. Natl. Cancer Inst. 89, 1360-5 (1997).

224. Yasui, K. et al. Characteristics of patients with nonalcoholic steatohepatitis who develop hepatocellular carcinoma. Clin. Gastroenterol. Hepatol. 9, 428-33; quiz e50 (2011).

225. Mittal, S. \& El-Serag, H. B. Epidemiology of hepatocellular carcinoma: consider the population. J. Clin. Gastroenterol. 47 Suppl, S2-6 (2013).

226. Jiang, W. et al. Dysbiosis gut microbiota associated with inflammation and impaired mucosal immune function in intestine of humans with non-alcoholic fatty liver disease. Sci. Rep. 5, 8096 (2015).

227. Mehal, W. Z. The Gordian Knot of dysbiosis, obesity and NAFLD. Nat. Rev. Gastroenterol. Hepatol. 10, 637-44 (2013).

228. Shen, J., Obin, M. S. \& Zhao, L. The gut microbiota, obesity and insulin resistance. Mol. Aspects Med. 34, 39-58 (2013).

229. Boursier, J. \& Diehl, A. M. Implication of gut microbiota in nonalcoholic fatty liver disease. PLoS Pathog. 11, e1004559 (2015).

230. Kleiner, D. E. et al. Design and validation of a histological scoring system for nonalcoholic fatty liver disease. Hepatology 41, 1313-21 (2005). 
231. Bedossa, P. et al. Histopathological algorithm and scoring system for evaluation of liver lesions in morbidly obese patients. Hepatology 56, 1751-9 (2012).

232. Nobili, V. et al. Retinol-binding protein 4: a promising circulating marker of liver damage in pediatric nonalcoholic fatty liver disease. Clin. Gastroenterol. Hepatol. 7, 575-9 (2009).

233. Younossi, Z. M. et al. A Novel Diagnostic Biomarker Panel for Obesity-related Nonalcoholic Steatohepatitis (NASH). Obes. Surg. 18, 1430-1437 (2008).

234. Palekar, N. A., Naus, R., Larson, S. P., Ward, J. \& Harrison, S. A. Clinical model for distinguishing nonalcoholic steatohepatitis from simple steatosis in patients with nonalcoholic fatty liver disease. Liver Int. 26, 151-6 (2006).

235. Harrison, S. A., Oliver, D., Arnold, H. L., Gogia, S. \& Neuschwander-Tetri, B. A. Development and validation of a simple NAFLD clinical scoring system for identifying patients without advanced disease. Gut 57, 1441-7 (2008).

236. Ratziu, V. et al. Liver fibrosis in overweight patients. Gastroenterology 118, 1117-23 (2000).

237. Speliotes, E. K. et al. Fatty liver is associated with dyslipidemia and dysglycemia independent of visceral fat: the Framingham Heart Study. Hepatology 51, 1979-87 (2010).

238. Hwang, S. et al. The effect of donor weight reduction on hepatic steatosis for living donor liver transplantation. Liver Transpl. 10, 721-5 (2004).

239. Malik, A., Cheah, P.-L., Hilmi, I. N., Chan, S. P. \& Goh, K.-L. Non-alcoholic fatty liver disease in Malaysia: a demographic, anthropometric, metabolic and histological study. J. Dig. Dis. 8, 5864 (2007).

240. Hamaguchi, M. et al. The metabolic syndrome as a predictor of nonalcoholic fatty liver disease. Ann. Intern. Med. 143, 722-8 (2005).

241. Fan, J.-G. et al. Prevalence of and risk factors for fatty liver in a general population of Shanghai, China. J. Hepatol. 43, 508-14 (2005).

242. Das, K. et al. Nonobese population in a developing country has a high prevalence of nonalcoholic fatty liver and significant liver disease. Hepatology 51, 1593-602 (2010).

243. Kwon, Y.-M. et al. Association of nonalcoholic fatty liver disease with components of metabolic syndrome according to body mass index in Korean adults. Am. J. Gastroenterol. 107, 1852-8 (2012).

244. el-Hassan, A. Y., Ibrahim, E. M., al-Mulhim, F. A., Nabhan, A. A. \& Chammas, M. Y. Fatty infiltration of the liver: analysis of prevalence, radiological and clinical features and influence on patient management. Br. J. Radiol. 65, 774-8 (1992).

245. Aboueisha, H. et al. A retrospective evaluation of causes of exempting living liver donors in an Egyptian centre. Arab J. Gastroenterol. 14, 10-3 (2013).

246. Strauss, R. S., Barlow, S. E. \& Dietz, W. H. Prevalence of abnormal serum aminotransferase values in overweight and obese adolescents. J. Pediatr. 136, 727-33 (2000). 
247. Zou, C. C., Liang, L., Hong, F., Fu, J. F. \& Zhao, Z. Y. Serum adiponectin, resistin levels and nonalcoholic fatty liver disease in obese children. Endocr. J. 52, 519-24 (2005).

248. Guzzaloni, G., Grugni, G., Minocci, A., Moro, D. \& Morabito, F. Liver steatosis in juvenile obesity: correlations with lipid profile, hepatic biochemical parameters and glycemic and insulinemic responses to an oral glucose tolerance test. Int. J. Obes. Relat. Metab. Disord. 24, 772-6 (2000).

249. Fu, J. et al. [Nonalcoholic steatohepatitis in obese children: the prevalence and possible mechanism]. Zhejiang Da Xue Xue Bao. Yi Xue Ban 35, 64-8 (2006).

250. Schwimmer, J. B. et al. Obesity, insulin resistance, and other clinicopathological correlates of pediatric nonalcoholic fatty liver disease. J. Pediatr. 143, 500-5 (2003).

251. Nobili, V. et al. NAFLD in children: a prospective clinical-pathological study and effect of lifestyle advice. Hepatology 44, 458-65 (2006).

252. Feldstein, A. E. et al. The natural history of non-alcoholic fatty liver disease in children: a follow-up study for up to 20 years. Gut 58, 1538-44 (2009).

253. Bedogni, G. et al. The Fatty Liver Index: a simple and accurate predictor of hepatic steatosis in the general population. BMC Gastroenterol. 6, 33 (2006).

254. Kotronen, A. et al. Prediction of non-alcoholic fatty liver disease and liver fat using metabolic and genetic factors. Gastroenterology 137, 865-72 (2009).

255. Poynard, T. et al. Performance of biomarkers FibroTest, ActiTest, SteatoTest, and NashTest in patients with severe obesity: meta analysis of individual patient data. PLoS One 7, e30325 (2012).

256. Angulo, P. et al. The NAFLD fibrosis score: a noninvasive system that identifies liver fibrosis in patients with NAFLD. Hepatology 45, 846-54 (2007).

257. Ratziu, V. et al. Diagnostic value of biochemical markers (FibroTest-FibroSURE) for the prediction of liver fibrosis in patients with non-alcoholic fatty liver disease. BMC Gastroenterol. 6, 6 (2006).

258. Shah, A. G. et al. Comparison of noninvasive markers of fibrosis in patients with nonalcoholic fatty liver disease. Clin. Gastroenterol. Hepatol. 7, 1104-12 (2009).

259. Adams, L. A. et al. Complex non-invasive fibrosis models are more accurate than simple models in non-alcoholic fatty liver disease. J. Gastroenterol. Hepatol. 26, 1536-43 (2011).

260. Guha, I. N. et al. Noninvasive markers of fibrosis in nonalcoholic fatty liver disease: Validating the European Liver Fibrosis Panel and exploring simple markers. Hepatology 47, 455-60 (2008). 
Highlighted references - wong

26. Wong, R. J. et al. Nonalcoholic steatohepatitis is the second leading etiology of liver disease among adults awaiting liver transplantation in the United States. Gastroenterology 148, 547-55 (2015).

Data from the UNOS/OPTN registry indicate that nonalcoholic steatohepatitis is the most rapidly growing indication for liver transplantation in USA in the last 10 years.

38. Kwok, R. et al. Screening diabetic patients for non-alcoholic fatty liver disease with controlled attenuation parameter and liver stiffness measurements: a prospective cohort study. Gut (2015). at http://www.ncbi.nlm.nih.gov/pubmed/25873639

This large cross-sectional study suggests that cirrhosis and advanced fibrosis are highly prevalent in diabetic patients, making type 2 diabetes a potential indication for fatty liver screening.

\section{Bugianesi}

I missed a reference (Fatty Liver Index, FLI, Table 4) hence ref. \# 254 changes and all the following shifts by one position. $I$ also changed the last reference to a more updated one

1. Bedogni G, Bellentani S, Miglioli L, Masutti F, Passalacqua M, Castiglione A, et al. The Fatty Liver Index: a simple and accurate predictor of hepatic steatosis in the general population. $B M C$ Gastroentero/ 6, 33 (2006). The first and most widely used index for the non-ultrasound detection of NAFLD.

2. Angulo, P. et al. The NAFLD fibrosis score: a noninvasive system that identifies liver fibrosis in patients with NAFLD. Hepatology 45, 846-54 (2007). The only non invasive index for the identification of NASH that has been validated both cross-sectionally and longitudinally.

3. EASL-ALEH Clinical Practice Guidelines: Non-invasive tests for evaluation of liver disease severity and prognosis. European Association for Study of Liver; Asociacion Latinoamericana para el Estudio del Higado. J Hepatol. 63, 237-64 (2015). The most recent guidelines for the non-invasive detection of liver fibrosis in liver disease of different aetiology, including NAFLD/NASH.

\section{Day}

17. Romeo, S. et al. Genetic variation in PNPLA3 confers susceptibility to nonalcoholic fatty liver disease. Nat. Genet. 40, 1461-5 (2008).

The is the first genome wide association study (GWAS) in NAFLD and convincingly identified the PNPLA3 gene as a risk factor for NAFLD 
98. Kozlitina, J. et al. Exome-wide association study identifies a TM6SF2 variant that confers susceptibility to nonalcoholic fatty liver disease. Nat. Genet. 46, 352-6 (2014).

This Exome-wide association study identified TM6SF2 as the second gene that confers susceptibility to NAFLD

Sookoian

114. Pirola, C. J. et al. Epigenetic modification of liver mitochondrial DNA is associated with histological severity of nonalcoholic fatty liver disease. Gut62, 1356-63 (2013).

This study provides first evidence of the role of mitochondrial epigenetics in human NAFLD.

130. Ahrens, M. et al. DNA methylation analysis in nonalcoholic fatty liver disease suggests distinct disease-specific and remodeling signatures after bariatric surgery. Cell Metab.18, 296-302 (2013).

This work illustrates the plasticity of the liver epigenome that can be modulated by therapeutic intervention.

\section{Maher}

Donnelly, K. L. et al. Sources of fatty acids stored in liver and secreted via lipoproteins in patients with nonalcoholic fatty liver disease. J. Clin. Invest. 115, 1343-51 (2005).

- \#55 and \#58 (duplicate refs) Donnelly et al, JCl 2005. This paper documents the relative contribution of dietary fat, DNL and adipose tissue lipolysis to hepatic lipid accumulation in humans.

61. Koliaki, C. et al. Adaptation of hepatic mitochondrial function in humans with non-alcoholic fatty liver is lost in steatohepatitis. Cell Metab. 21, 739-46 (2015).

- \# $\underline{61}$ Koliaki et al, Cell Metab 2015. This paper documents that hepatic steatosis is marked by a compensatory increase in mitochondrial activity, but this compensation is lost as NASH develops.

60. Schnabl, B. \& Brenner, D. A. Interactions between the intestinal microbiome and liver diseases. Gastroenterology 146, 1513-24 (2014).

- \# 60 Schnabl and Brenner, Gastroenterology 2014. This review documents the role of gut dysbiosis in the pathogenesis of NAFLD. 
78. Mehal, W. Z. The inflammasome in liver injury and non-alcoholic fatty liver disease. Dig. Dis. 32, 507-15 (2014).

- \# $\underline{78}$ Mehal, WZ, Dig Dis Sci 2014. This review describes the role of inflammasome activation in the pathogenesis of NAFLD.

\section{Rinella}

Selected references:

1. Vilar-Gomez, E. et al. Weight Loss via Lifestyle Modification Significantly Reduces Features of Nonalcoholic Steatohepatitis. Gastroenterology 149, 367-378.e5 (2015).

Largest trial of lifestyle intervention to date which demonstrated resolution of NASH and improvement or stabilization of fibrosis in those that lost $>7 \%$ body weight.

2. Lassailly G, Caiazzo R, Buob D, Pigeyre M, Verkindt H, Labreuche J, Raverdy V, Leteurtre E, Dharancy S, Louvet A, Romon M, Duhamel A, Pattou F, Mathurin P. Gastroenterology. 2015 Aug;149(2):379-88. doi: 10.1053/j.gastro.2015.04.014. Epub 2015 Apr 25. PMID: 25917783

First large study to show that one year after bariatric surgery, NASH resolved in $85 \%$ of patients and approximately one third had an improvement in fibrosis, which had not been previously demonstrated.

3. Welzel, T. M. et al. Population-attributable fractions of risk factors for hepatocellular carcinoma in the United States. Am. J. Gastroenterol. 108, 1314-21 (2013).

Important data from the SEER database demonstrating that while the risk of HCC in patients with NASH m99ay be lower than other diseases (i.e HCV), its high prevalence makes the population attributable risk of HCC in NASH is nearly $40 \%$. 